\title{
Benzannulation of Triynes Initiated by an Alder-Ene Reaction and Subsequent Trifluoromethylthiolate Addition
}

\author{
Rajdip Karmakar, ${ }^{\dagger}$ Phani Mamidipalli, ${ }^{\dagger}$ Ryan M. Salzman, ${ }^{\dagger}$ Seongwon Hong, ${ }^{\dagger}$ Sang \\ Young Yun, ${ }^{+}$Wei Guo, ${ }^{\ddagger}$ Yuanzhi Xia, ${ }^{* \ddagger}$ and Daesung Lee*' \\ ${ }^{\dagger}$ Department of Chemistry, University of Illinois at Chicago, 845 West Taylor Street, \\ Chicago, Illinois 60607 \\ ${ }^{\ddagger}$ College of Chemistry and Materials Engineering, Wenzhou University, Wenzhou, \\ Zhejiang Province 325035, P.R. China \\ dsunglee@uic.edu
}

\section{Contents}

General Information

S1-S2

Experimental Details and Characterization Data

S2-S8

${ }^{1} \mathrm{H},{ }^{13} \mathrm{C}$ and ${ }^{19} \mathrm{~F}$ Spectra

S9-S23

Computational Study

S24-S28

General Information: Reactions were carried out in oven-dried glassware unless otherwise noted. Compounds were purchased from Aldrich or Acros or $\mathrm{TCl}$ America or Oakwood Chemicals unless otherwise noted. Toluene, acetonitrile and dichloromethane were distilled over calcium hydride $\left(\mathrm{CaH}_{2}\right)$ under nitrogen atmosphere. THF was distilled over sodium-benzophenone ketyl under nitrogen atmosphere. Column chromatography was performed using silica gel $60 \AA$ (32-63 mesh) purchased from Silicycle Inc. Analytical thin layer chromatography (TLC) was performed on $0.25 \mathrm{~mm}$ E. Merck precoated silica gel 60 (particle size 0.040-0.063 $\mathrm{mm}$ ). Yields refer to chromatographically and spectroscopically pure compounds unless otherwise stated. ${ }^{1} \mathrm{H} N M R$ and ${ }^{13} \mathrm{C}$ NMR spectra were recorded on a Bruker 
AV-500 spectrometer. ${ }^{19} \mathrm{~F}$ NMR spectrum was recorded in Varian Mercury-Vx-300 spectrometer. ${ }^{1} \mathrm{H}$ NMR chemical shifts $(\delta)$ are reported in parts per million (ppm) downfield of TMS and are referenced relative to the residual proteated solvent peak $\left(\mathrm{CDCl}_{3}(7.26 \mathrm{ppm})\right) .{ }^{13} \mathrm{C}$ chemical shifts $(\delta)$ are reported in parts per million downfield of TMS and are referenced to the carbon resonance of the solvent $\left(\mathrm{CDCl}_{3}\right.$ (77.2 ppm)). Multiplicities are indicated by s (singlet), $d$ (doublet), $t$ (triplet), q (quartet), quin (quintet), sext (sextet) or $\mathrm{m}$ (multiplet). ${ }^{1} \mathrm{H}$ NMR signals that fall within a ca. 0.3 ppm range are generally reported as a multiplet, with a range of chemical shift values corresponding to the peak or center of the peak. Coupling constants, J, are reported in $\mathrm{Hz}$ (Hertz). Electrospray ionization (ESI) mass spectra were recorded on a Waters Micromass Q-Tof Ultima in the University of Illinois at Urbana-Champaign. Electron impact (EI) mass spectra and Chemical lonization (CI) mass spectra were obtained using a Micromass 70-VSE in the University of Illinois at Urbana-Champaign.

\section{Experimental Details}

\section{General procedure for the generation of trifluoromethylthiolated arene products}

To a Schlenk tube containing a mixture of a substrate (0.1 mmol, 1 equiv) and $\mathrm{AgSCF}_{3}$ (0.15 mmol, 1.5 equiv) in dry toluene ( $3 \mathrm{~mL}$ ) was added $20 \mu \mathrm{L} \mathrm{CF}_{3} \mathrm{CH}_{2} \mathrm{OH}$ and the reaction mixture was stirred at $90{ }^{\circ} \mathrm{C}$ for $5 \mathrm{~h}$ (or until completion). The reaction mixture was transferred to a round-bottom flask, concentrated and subjected to column chromatography, using ethyl acetate-hexane mixture as an eluent, to get pure products.

\section{Preparation of Starting Materials}

$\mathrm{AgSCF}_{3}$ was prepared following the reported procedure. ${ }^{1}$

Starting materials (triynes) were prepared following the general procedures reported previously. $^{2}$ 


\section{NMR Study for the Aromatization of Nonaromatic Compound}

A $\mathrm{CD}_{3} \mathrm{CN}$ solution of nonaromatic compound $2 \mathrm{~m}^{\prime}$ was taken in a NMR reaction tube and heated at $90{ }^{\circ} \mathrm{C}$. NMR monitoring shows its clean conversion to the corresponding aromatic compound $2 \mathrm{~m}$. Below is the comparison of the NMRs at several interval which depicts the fast progress of the aromatization reaction.<smiles>C=CCOCCC1C(C(F)(F)F)=C2C(=O)N([As])CC2=C/C1=C\[I-](F)(F)F</smiles><smiles>C=CCOCCc1c(C[In])cc2c(c1C(F)(F)F)C(=O)N([As])C2</smiles>
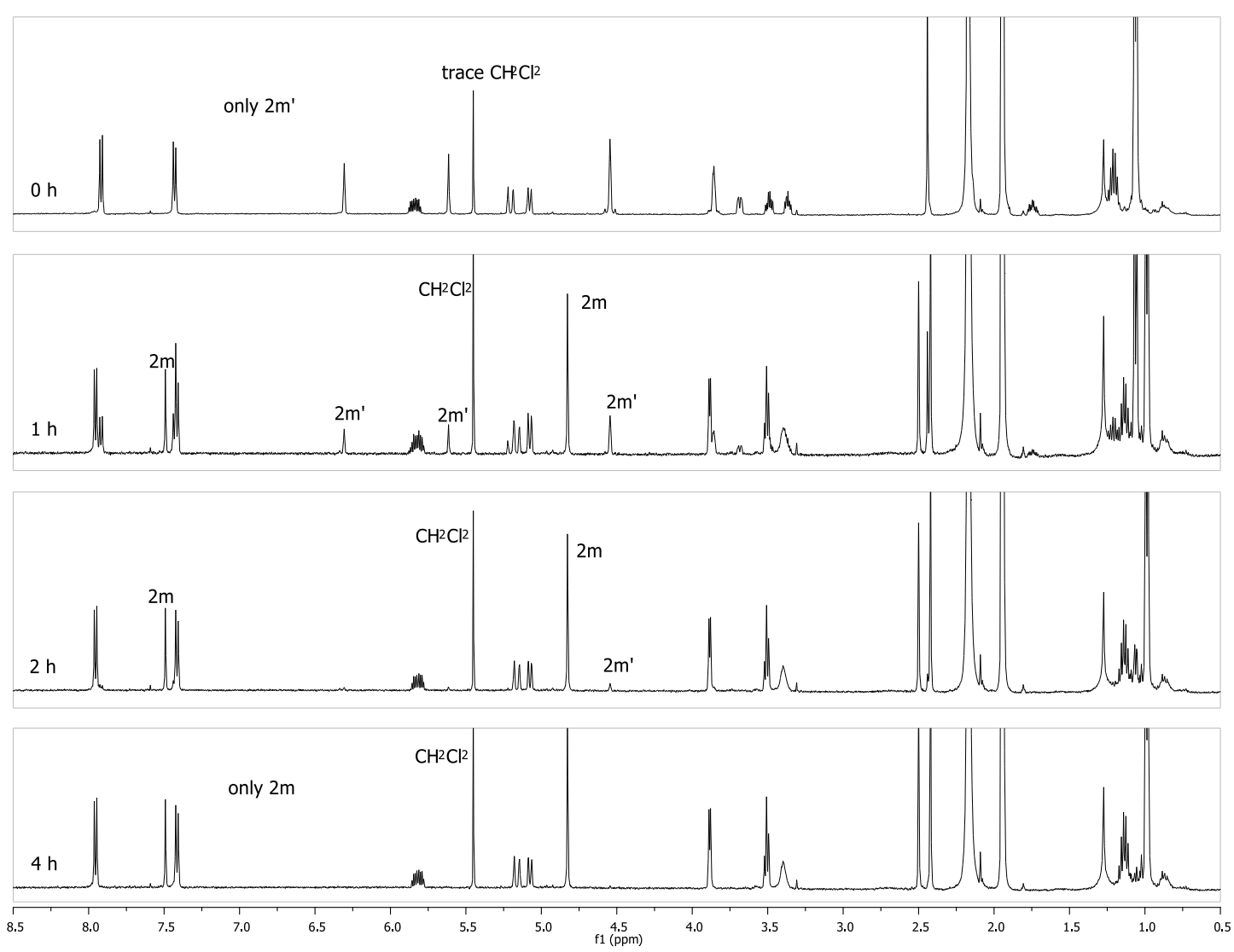


\section{Characterization Data of the Products}

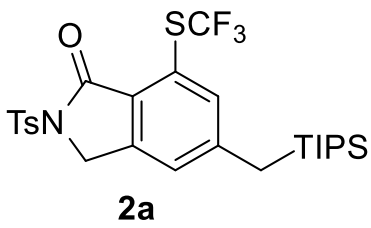

(m, 3H), 1.01-1.00 (m, 18H); $\left.{ }^{13} \mathrm{C} \mathrm{NMR} \mathrm{(CDCl}, 125 \mathrm{MHz}\right): \delta 164.4,150.6,145.3,143.0$, $135.3,131.9,129.8,128.3,128.1,126.4,124.7,123.0,48.9,21.7,21.0,18.5,10.9 ;{ }^{19} \mathrm{~F}$ NMR $\left(\mathrm{CDCl}_{3}, 300 \mathrm{MHz}\right) \delta-40.58$. HRMS (ESI) calcd for $\mathrm{C}_{26} \mathrm{H}_{35} \mathrm{~F}_{3} \mathrm{NO}_{3} \mathrm{~S}_{2} \mathrm{Si}^{+}[\mathrm{M}+\mathrm{H}]^{+}$ 558.1774 , found 558.1776 .<smiles>Cc1c(C[18OH])cc2c(c1C(F)(F)F)C(=O)N([As])C2</smiles>

2b

This compound was isolated in $71 \%$ yield $(39.5 \mathrm{mg}$ ) after purification by column chromatography. ${ }^{1} \mathrm{H}$ NMR $\left(\mathrm{CDCl}_{3}, 500\right.$ $\mathrm{MHz}):$ $\delta 8.04-8.02(\mathrm{~m}, 2 \mathrm{H}), 7.38(\mathrm{~s}, 1 \mathrm{H}), 7.35-7.34(\mathrm{~m}, 2 \mathrm{H})$, $7.14(\mathrm{~s}, 1 \mathrm{H}), 4.82(\mathrm{~s}, 2 \mathrm{H}), 2.42(\mathrm{~s}, 3 \mathrm{H}), 2.33(\mathrm{~s}, 2 \mathrm{H}), 1.10-1.03$ 
$\mathrm{MHz}): \delta 168.7,151.0,148.7,133.4,130.5,128.1,126.0,122.1,121.3,68.5,20.9$, $18.5,10.9 ;{ }^{19} \mathrm{~F} \mathrm{NMR}\left(\mathrm{CDCl}_{3}, 300 \mathrm{MHz}\right) \delta-40.77$. HRMS (ESI) calcd for $\mathrm{C}_{19} \mathrm{H}_{28} \mathrm{~F}_{3} \mathrm{O}_{2} \mathrm{SSi}^{+}$ $[\mathrm{M}+\mathrm{H}]^{+}$405.1526, found 405.1529 .

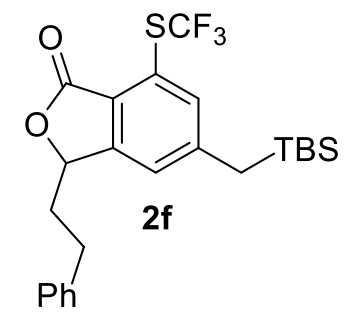

This compound was isolated in $67 \%$ yield $(31.2 \mathrm{mg})$ after purification by column chromatography. ${ }^{1} \mathrm{H}$ NMR $\left(\mathrm{CDCl}_{3}, 500\right.$ $\mathrm{MHz}): \delta 7.34(\mathrm{~s}, 1 \mathrm{H}), 7.32-7.29(\mathrm{~m}, 2 \mathrm{H}), 7.23-7.21(\mathrm{~m}, 3 \mathrm{H}), 7.00$ (s, $1 \mathrm{H}), 5.39-5.36(\mathrm{~m}, 1 \mathrm{H}), 2.87-2.75(\mathrm{~m}, 2 \mathrm{H}), 2.33-2.27(\mathrm{~m}$, $1 \mathrm{H}), 2.27(\mathrm{~s}, 2 \mathrm{H}), 2.05-1.98(\mathrm{~m}, 1 \mathrm{H}), 0.93(\mathrm{~s}, 9 \mathrm{H}),-0.09(\mathrm{~s}, 6 \mathrm{H})$;

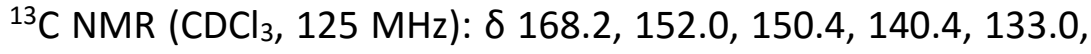
130.6, 128.6, 128.5, 128.3, 128.1, 126.4, 126.1, 121.6, 121.4, 79.1, 36.6, 31.1, 26.4, 24.5, 16.9, $-6.7 ;{ }^{19} \mathrm{~F} \mathrm{NMR}\left(\mathrm{CDCl}_{3}, 300 \mathrm{MHz}\right) \delta-40.78$. HRMS (ESI) calcd for $\mathrm{C}_{24} \mathrm{H}_{30} \mathrm{~F}_{3} \mathrm{O}_{2} \mathrm{SSi}^{+}[\mathrm{M}+\mathrm{H}]^{+}$467.1682, found 467.1689.

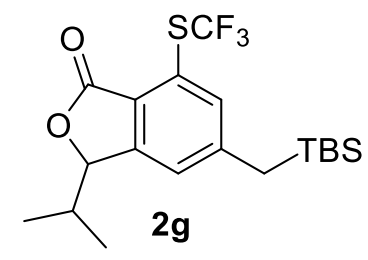

This compound was isolated in $59 \%$ yield $(24.0 \mathrm{mg})$ after purification by column chromatography. ${ }^{1} \mathrm{H} \mathrm{NMR}\left(\mathrm{CDCl}_{3}, 500\right.$ $\mathrm{MHz}): \delta 7.34(\mathrm{~s}, 1 \mathrm{H}), 7.03(\mathrm{~s}, 1 \mathrm{H}), 5.28(\mathrm{~d}, 1 \mathrm{H}, J=3.0 \mathrm{~Hz}), 2.28$ $(\mathrm{s}, 2 \mathrm{H}), 2.27-2.22(\mathrm{~m}, 1 \mathrm{H}), 1.13(\mathrm{~d}, 3 \mathrm{H}, J=7.0 \mathrm{~Hz}), 0.92(\mathrm{~s}, 9 \mathrm{H})$, $0.76(\mathrm{~d}, 3 \mathrm{H}, J=7.0 \mathrm{~Hz}),-0.08(\mathrm{~s}, 3 \mathrm{H}),-0.10(\mathrm{~s}, 3 \mathrm{H}) ;{ }^{13} \mathrm{C} N \mathrm{NMR}$ $\left(\mathrm{CDCl}_{3}, 125 \mathrm{MHz}\right): \delta 168.4,151.2,150.0,132.8,130.6,128.1,126.0,122.2,121.8$, $84.3,32.4,26.4,24.5,18.8,16.9,15.3,-6.67,-6.7 ;{ }^{19} \mathrm{~F} \mathrm{NMR}\left(\mathrm{CDCl}_{3}, 300 \mathrm{MHz}\right) \delta$. HRMS (ESI) calcd for $\mathrm{C}_{19} \mathrm{H}_{28} \mathrm{~F}_{3} \mathrm{O}_{2} \mathrm{SSi}^{+}[\mathrm{M}+\mathrm{H}]^{+}$405.1526, found 405.1527.

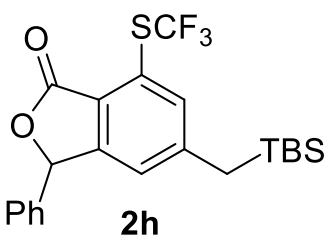

This compound was isolated in $64 \%$ yield $(28.0 \mathrm{mg})$ after purification by column chromatography. ${ }^{1} \mathrm{H}$ NMR $\left(\mathrm{CDCl}_{3}, 500\right.$ $\mathrm{MHz}): \delta 7.39-7.37(\mathrm{~m}, 4 \mathrm{H}), 7.25-7.24(\mathrm{~m}, 2 \mathrm{H}), 6.90(\mathrm{~s}, 1 \mathrm{H})$, $6.30(\mathrm{~s}, 1 \mathrm{H}), 2.23(\mathrm{~d}, 2 \mathrm{H}, \mathrm{J}=3.0 \mathrm{~Hz}), 0.88(\mathrm{~s}, 9 \mathrm{H}),-0.12(\mathrm{~s}, 3 \mathrm{H})$, $-0.19(\mathrm{~s}, 3 \mathrm{H}) ;{ }^{13} \mathrm{C} \mathrm{NMR}\left(\mathrm{CDCl}_{3}, 125 \mathrm{MHz}\right): \delta$ 168.1, 151.9, 150.7, 136.1, 133.3, 129.5, 129.0, 127.1, 125.9, 122.7, 121.1, 81.5, 26.4, 24.51, 16.8, -6.7, $-6.9 ;{ }^{19} \mathrm{~F} \mathrm{NMR}\left(\mathrm{CDCl}_{3}, 300 \mathrm{MHz}\right) \delta-40.80$. HRMS (ESI) calcd for $\mathrm{C}_{22} \mathrm{H}_{26} \mathrm{~F}_{3} \mathrm{O}_{2} \mathrm{SSi}^{+}[\mathrm{M}+\mathrm{H}]^{+}$ 439.1369, found 439.1378 . 


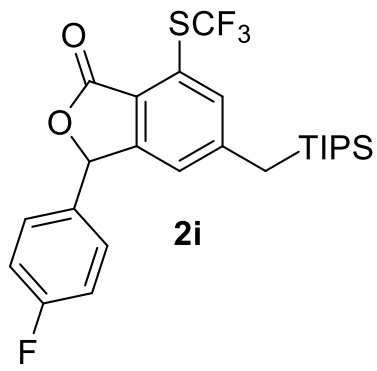

This compound was isolated in $35 \%$ yield $(17.4 \mathrm{mg})$ after purification by column chromatography. ${ }^{1} \mathrm{H} \mathrm{NMR}\left(\mathrm{CDCl}_{3}, 500\right.$ $\mathrm{MHz}): \delta 7.50(\mathrm{~s}, 1 \mathrm{H}), 7.26-7.23(\mathrm{~m}, 2 \mathrm{H}), 7.10(\mathrm{t}, 2 \mathrm{H}, J=8.5$ $\mathrm{Hz}), 6.98(\mathrm{~s}, 1 \mathrm{H}), 6.30(\mathrm{~s}, 1 \mathrm{H}), 2.35(\mathrm{~s}, 2 \mathrm{H}), 1.07-0.99(\mathrm{~m}, 3 \mathrm{H})$, 0.98-0.96 (m, 18H); ${ }^{13} \mathrm{C} \mathrm{NMR}\left(\mathrm{CDCl}_{3}, 125 \mathrm{MHz}\right): \delta 167.9$, $164.3,162.3,151.6,151.4,133.2,131.98,131.96,130.5$, 129.1, 129.0, 128.1, 126.2, 126.18, 122.9, 120.8, 116.1, 116.0, 80.8, 21.1, 18.4, 18.4, 10.8; ${ }^{19} \mathrm{~F} \mathrm{NMR}\left(\mathrm{CDCl}_{3}, 300 \mathrm{MHz}\right) \delta-38.36,-109.61$. HRMS (ESI) calcd for $\mathrm{C}_{25} \mathrm{H}_{31} \mathrm{~F}_{4} \mathrm{O}_{2} \mathrm{SSi}^{+}[\mathrm{M}+\mathrm{H}]^{+}$521.1564, found 521.1571.

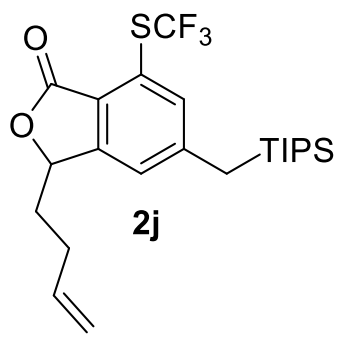

This compound was isolated in $67 \%$ yield $(30.7 \mathrm{mg}$ ) after purification by column chromatography. ${ }^{1} \mathrm{H}$ NMR $\left(\mathrm{CDCl}_{3}, 500\right.$ $\mathrm{MHz}): \delta 7.44(\mathrm{~s}, 1 \mathrm{H}), 7.11(\mathrm{~s}, 1 \mathrm{H}), 5.85-5.77(\mathrm{~m}, 1 \mathrm{H}), 5.40(\mathrm{dd}$, $1 \mathrm{H}, J=8.0,3.5 \mathrm{~Hz}), 5.08-5.02(\mathrm{~m}, 2 \mathrm{H}), 2.37(\mathrm{~s}, 2 \mathrm{H}), 2.30-2.15$ $(\mathrm{m}, 2 \mathrm{H}), 2.13-2.06(\mathrm{~m}, 1 \mathrm{H}), 1.84-1.76(\mathrm{~m}, 1 \mathrm{H}), 1.13-1.01(\mathrm{~m}$, $21 \mathrm{H}) ;{ }^{13} \mathrm{C} \mathrm{NMR}\left(\mathrm{CDCl}_{3}, 125 \mathrm{MHz}\right): \delta$ 168.2, 152.0, 150.9, 136.6, $132.8,130.6,126.2,121.7,121.4,116.1,79.3,33.9,28.8,21.0,18.5,10.9 ;{ }^{19} \mathrm{~F} \mathrm{NMR}$ $\left(\mathrm{CDCl}_{3}, 300 \mathrm{MHz}\right) \delta-40.72$. HRMS (ESI) calcd for $\mathrm{C}_{23} \mathrm{H}_{34} \mathrm{~F}_{3} \mathrm{O}_{2} \mathrm{SSi}^{+}[\mathrm{M}+\mathrm{H}]^{+}$459.1995, found 459.2003.

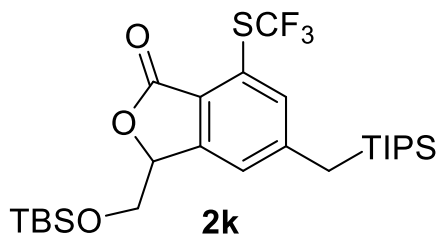

This compound was isolated in 59\% yield ( $32.4 \mathrm{mg}$ ) after purification by column chromatography. ${ }^{1} \mathrm{H} \mathrm{NMR}\left(\mathrm{CDCl}_{3}\right.$, $500 \mathrm{MHz}$ ): $\delta 7.46(\mathrm{~s}, 1 \mathrm{H}), 7.25(\mathrm{~s}, 1 \mathrm{H}), 5.34(\mathrm{t}, 1 \mathrm{H}, J=4.8$ $\mathrm{Hz}), 4.02(\mathrm{dd}, 1 \mathrm{H}, J=11.0,4.0 \mathrm{~Hz}$ ), 3.89 (dd, $1 \mathrm{H}, J=11.0$, $5.2 \mathrm{~Hz}), 2.36(\mathrm{~s}, 2 \mathrm{H}), 1.11-1.06(\mathrm{~m}, 3 \mathrm{H}), 1.02-1.01(\mathrm{~m}$, $18 \mathrm{H}), 0.82(\mathrm{~s}, 9 \mathrm{H}), 0.044(\mathrm{~s}, 3 \mathrm{H}), 0.036(\mathrm{~s}, 3 \mathrm{H}) ;{ }^{13} \mathrm{C} \mathrm{NMR}\left(\mathrm{CDCl}_{3}, 125 \mathrm{MHz}\right): \delta$ 168.0, 150.6, 150.1, 133.6, 130.6, 128.1, 125.6, 122.7, 122.1, 79.6, 63.9, 25.6, 20.9, 18.6, 18.5, 10.9, $-5.6 ;{ }^{19} \mathrm{~F} \mathrm{NMR}\left(\mathrm{CDCl}_{3}, 300 \mathrm{MHz}\right) \delta-40.78$. HRMS (ESI) calcd for $\mathrm{C}_{26} \mathrm{H}_{44} \mathrm{~F}_{3} \mathrm{O}_{3} \mathrm{SSi}_{2}{ }^{+}[\mathrm{M}+\mathrm{H}]^{+}$549.2496, found 549.2501.

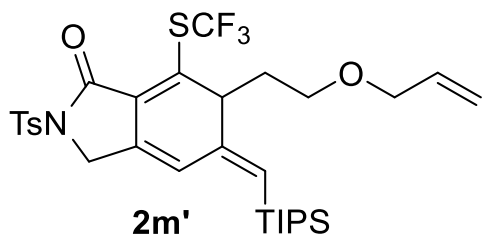

This compound was isolated in $21 \%$ yield $(13.5 \mathrm{mg})$ after purification by column chromatography. ${ }^{1} \mathrm{H}$ NMR $\left(\mathrm{CDCl}_{3}, 500 \mathrm{MHz}\right): \delta 8.01(\mathrm{~m}, 2 \mathrm{H}), 7.38(\mathrm{~m}, 2 \mathrm{H}), 6.19$ $(\mathrm{m}, 1 \mathrm{H}), 5.90-5.83(\mathrm{~m}, 1 \mathrm{H}), 5.60(\mathrm{~s}, 1 \mathrm{H}), 5.27-5.23(\mathrm{~m}$, $1 \mathrm{H}), 5.16-5.13(\mathrm{~m}, 1 \mathrm{H}), 4.62-4.49(\mathrm{~m}, 2 \mathrm{H}), 3.95-3.88$ 
(m, 2H), $3.73(\mathrm{dd}, 1 \mathrm{H}, J=10.0,7.5 \mathrm{~Hz}), 3.50(\mathrm{dt}, 1 \mathrm{H}, J=9.5,5.5 \mathrm{~Hz}), 3.41-3.37(\mathrm{~m}$, $1 \mathrm{H}), 2.47(\mathrm{~s}, 3 \mathrm{H}), 2.07-2.01(\mathrm{~m}, 1 \mathrm{H}), 1.75-1.68(\mathrm{~m}, 1 \mathrm{H}), 1.21-1.14(\mathrm{~m}, 3 \mathrm{H}), 1.09-1.07$ $(\mathrm{m}, 18 \mathrm{H}) ;{ }^{13} \mathrm{C} \mathrm{NMR}\left(\mathrm{CDCl}_{3}, 125 \mathrm{MHz}\right): \delta 162.8,150.2,145.6,134.9,134.7,134.6$, 129.8, 129.0, 128.41, 128.39, 128.37, 121.0, 116.4, 71.6, 64.0, 50.4, 48.5, 34.4, 29.7, 21.7, 18.9, 18.8, 12.2; ${ }^{19} \mathrm{~F} \mathrm{NMR}\left(\mathrm{CDCl}_{3}, 300 \mathrm{MHz}\right) \delta-37.21$. HRMS (ESI) calcd for $\mathrm{C}_{31} \mathrm{H}_{43} \mathrm{~F}_{3} \mathrm{NO}_{4} \mathrm{~S}_{2} \mathrm{Si}^{+}[\mathrm{M}+\mathrm{H}]^{+}$642.2349, found 642.2347.

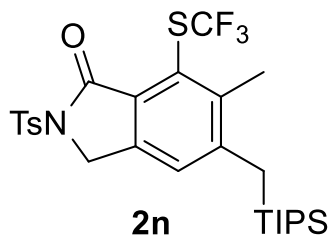

This compound was isolated in $16 \%$ yield $(9.1 \mathrm{mg})$ after purification by column chromatography. ${ }^{1} \mathrm{H}$ NMR $\left(\mathrm{CDCl}_{3}, 500\right.$ $\mathrm{MHz}):$ 8 8.05-8.03 (m, 2H), 7.34-7.32 (m, 2H), $7.29(\mathrm{~s}, 1 \mathrm{H}), 4.76$ $(\mathrm{s}, 2 \mathrm{H}), 2.61(\mathrm{~s}, 3 \mathrm{H}), 2.42(\mathrm{~s}, 3 \mathrm{H}), 2.37(\mathrm{~s}, 2 \mathrm{H}), 1.13-1.06(\mathrm{~m}$, $3 \mathrm{H}), 0.10-0.98(\mathrm{~m}, 18 \mathrm{H}) ;{ }^{13} \mathrm{C} \mathrm{NMR}\left(\mathrm{CDCl}_{3}, 125 \mathrm{MHz}\right): \delta 163.9$, $149.8,145.1,144.4,140.4,135.4,129.7,128.9,128.3,125.9,122.3,47.6,21.7,19.2$, $18.5,18.3,11.5$.

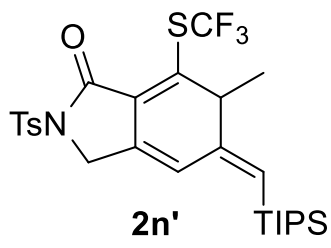

This compound was isolated in $49 \%$ yield $(28 \mathrm{mg}$ ) along with $\mathbf{2 n}$ (16\% yield) after purification by column chromatography. ${ }^{1} \mathrm{H}$ NMR $\left(\mathrm{CDCl}_{3}, 500 \mathrm{MHz}\right): \delta 7.98(\mathrm{~m}, 2 \mathrm{H}), 7.36(\mathrm{~m}, 2 \mathrm{H}), 6.19(\mathrm{~s}$, $1 \mathrm{H}), 5.58(\mathrm{~s}, 1 \mathrm{H}), 4.61(\mathrm{~d}, 1 \mathrm{H}, \mathrm{J}=15.5 \mathrm{~Hz}), 4.51(\mathrm{~d}, 1 \mathrm{H}, \mathrm{J}=15.5$ $\mathrm{Hz}), 3.54(\mathrm{q}, 1 \mathrm{H}, \mathrm{J}=7.0 \mathrm{~Hz}), 2.44(\mathrm{~s}, 3 \mathrm{H}), 1.35(\mathrm{~d}, 3 \mathrm{H}, \mathrm{J}=7.0 \mathrm{~Hz})$, $1.18-1.10(\mathrm{~m}, 3 \mathrm{H}), 1.05(\mathrm{~d}, 18 \mathrm{H}, \mathrm{J}=7.0 \mathrm{~Hz}) ;{ }^{13} \mathrm{C} \mathrm{NMR}\left(\mathrm{CDCl}_{3}, 125 \mathrm{MHz}\right): \delta 163.1$, 154.1, 145.6, 143.6, 134.9, 131.6, 130.1, 129.8, 128.5, 128.4, 127.6, 125.2, 120.6, 110.0, 48.6, 48.1, 26.6, 21.7, 18.82, 18.79, 12.3; $\left.{ }^{19} \mathrm{~F} \mathrm{NMR} \mathrm{(CDCl} 3,300 \mathrm{MHz}\right) \delta-37.26$. HRMS (EEI) calcd for $\mathrm{C}_{36} \mathrm{H}_{48} \mathrm{~F}_{3} \mathrm{~N}_{2} \mathrm{O}_{5} \mathrm{~S}_{3} \mathrm{Si}^{+}[\mathrm{M}+\mathrm{H}]^{+}$572.1931, found 572.1932 .<smiles>CN(C)CCC1C(=C[In])C=C2CN([Te])C(=O)C2=C1C(F)(F)F</smiles>

This compound was isolated in $25 \%$ yield (19.2 mg) after purification by column chromatography. ${ }^{1} \mathrm{H} \mathrm{NMR}\left(\mathrm{CDCl}_{3}\right.$, $500 \mathrm{MHz}): \delta 8.00(\mathrm{~m}, 2 \mathrm{H}), 7.61(\mathrm{~m}, 2 \mathrm{H}), 7.38(\mathrm{~m}, 2 \mathrm{H}), 7.30$ $(\mathrm{m}, 2 \mathrm{H}), 6.23(\mathrm{~m}, 1 \mathrm{H}), 5.67(\mathrm{~m}, 1 \mathrm{H}), 4.64-4.51(\mathrm{~m}, 2 \mathrm{H}), 3.49$ $(\mathrm{dd}, 1 \mathrm{H}, J=8.5,3.5 \mathrm{~Hz}), 3.06-2.96(\mathrm{~m}, 2 \mathrm{H}), 2.69(\mathrm{~s}, 3 \mathrm{H})$, $2.46(\mathrm{~s}, 3 \mathrm{H}), 2.44(\mathrm{~s}, 3 \mathrm{H}), 1.90-1.79(\mathrm{~m}, 2 \mathrm{H}), 1.22-1.15(\mathrm{~m}, 3 \mathrm{H}), 1.10-1.07(\mathrm{~m}, 18 \mathrm{H})$; ${ }^{13} \mathrm{C} \mathrm{NMR}\left(\mathrm{CDCl}_{3}, 125 \mathrm{MHz}\right): \delta 162.6,150.2,145.6,143.4,135.4,134.8,134.5,129.9$, 129.8, 129.7, 129.6, 129.2, 128.4, 127.3, 127.29, 121.3, 51.3, 48.5, 45.8, 34.9, 32.2, 21.8, 21.5, 18.9, 18.8, 12.2; $\left.{ }^{19} \mathrm{~F} \mathrm{NMR} \mathrm{(CDCl} 3,300 \mathrm{MHz}\right) \delta-39.55$. HRMS (ESI) calcd for $\mathrm{C}_{36} \mathrm{H}_{48} \mathrm{~F}_{3} \mathrm{~N}_{2} \mathrm{O}_{5} \mathrm{~S}_{3} \mathrm{Si}^{+}[\mathrm{M}+\mathrm{H}]^{+}$769.2441, found 769.2428. 


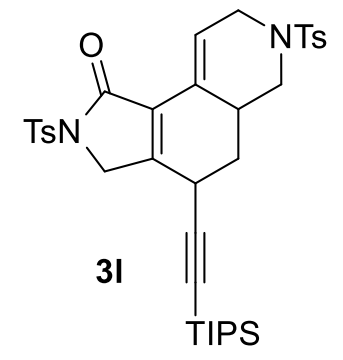

This compound was isolated in $95 \%$ yield $(64.5 \mathrm{mg})$ after purification by column chromatography. ${ }^{1} \mathrm{H}$ NMR $\left(\mathrm{CDCl}_{3}, 500\right.$ $M H z): \delta 7.91-7.89(m, 2 H), 7.66-7.64(m, 2 H), 7.32-7.30(m$, $4 \mathrm{H}), 6.69-6.68(\mathrm{~m}, 1 \mathrm{H}), 4.49(\mathrm{~d}, 1 \mathrm{H}, J=19.0 \mathrm{~Hz}), 4.33(\mathrm{~d}, 1 \mathrm{H}, J=$ $19.0 \mathrm{~Hz}$ ), 4.14-4.11 (m, 1H), 3.97 (dd, $1 \mathrm{H}, J=11.0,5.0 \mathrm{~Hz}), 3.51$ $(\mathrm{d}, 1 \mathrm{H}, J=5.0 \mathrm{~Hz}), 3.30-3.26(\mathrm{~m}, 1 \mathrm{H}), 2.84(\mathrm{~m}, 1 \mathrm{H}), 2.42(\mathrm{~s}, 6 \mathrm{H})$, $2.18(\mathrm{t}, 1 \mathrm{H}, J=11.0 \mathrm{~Hz}$ ), $1.99(\mathrm{dd}, 1 \mathrm{H}, J=13.0,2.5 \mathrm{~Hz}$ ), $1.40(\mathrm{dt}, 1 \mathrm{H}, J=13.0,5.5 \mathrm{~Hz}$ ),

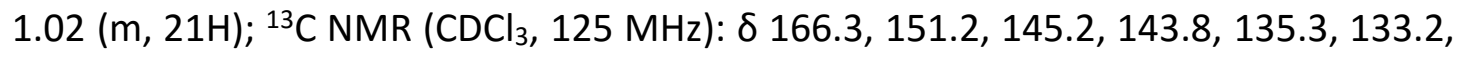
129.84, 129.78, 129.7, 129.6, 127.9, 127.8, 127.6, 126.6, 126.1, 121.3, 104.4, 85.1, 50.5, 47.4, 45.1, 31.9, 31.1, 27.5, 21.7, 21.5, 18.6, 11.1. HRMS (ESI) calcd for $\mathrm{C}_{36} \mathrm{H}_{47} \mathrm{~N}_{2} \mathrm{O}_{5} \mathrm{~S}_{2} \mathrm{Si}^{+}[\mathrm{M}+\mathrm{H}]^{+}$679.2690, found 679.2703.

\section{References:}

1. Teverovskiy, G.; Surry, D. S.; Buchwald, S. L. Angew. Chem. Int. Ed. 2011, 50, 7312.

2. Karmakar, R. Yun, S. Y.; Chen, J.; Xia, Y.; Lee, D. Angew. Chem. Int. Ed. 2015, $54,6582$. 

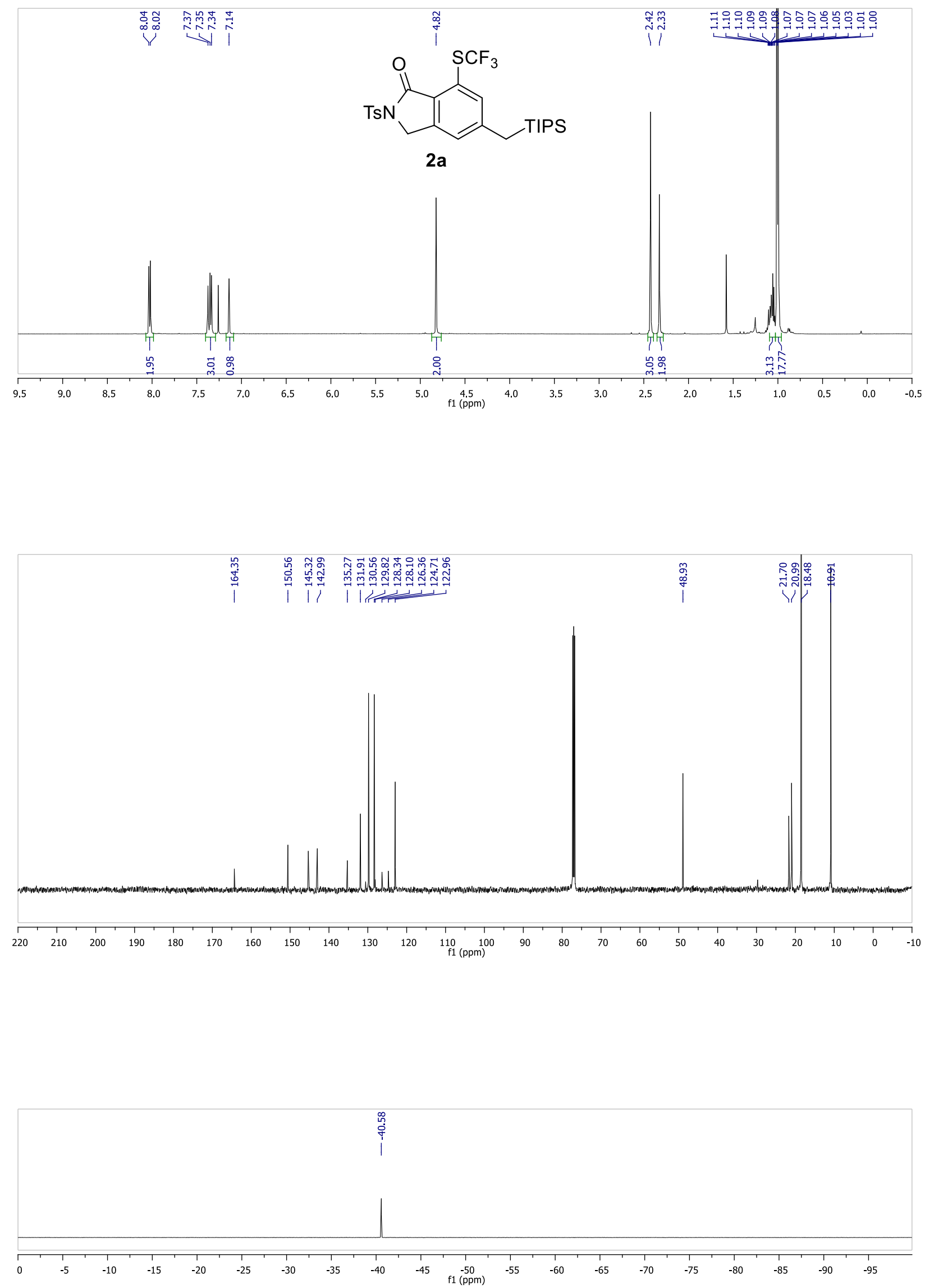

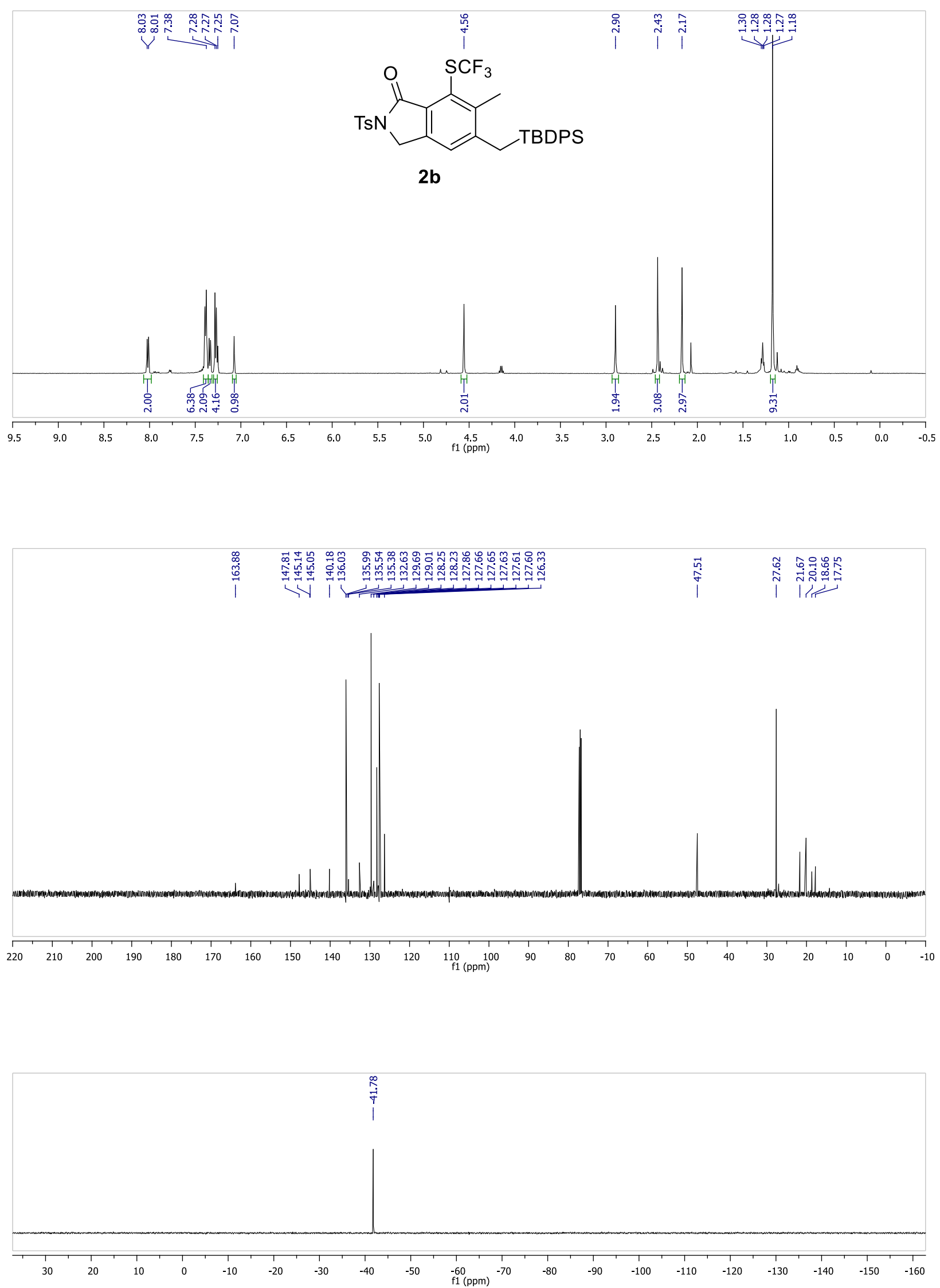

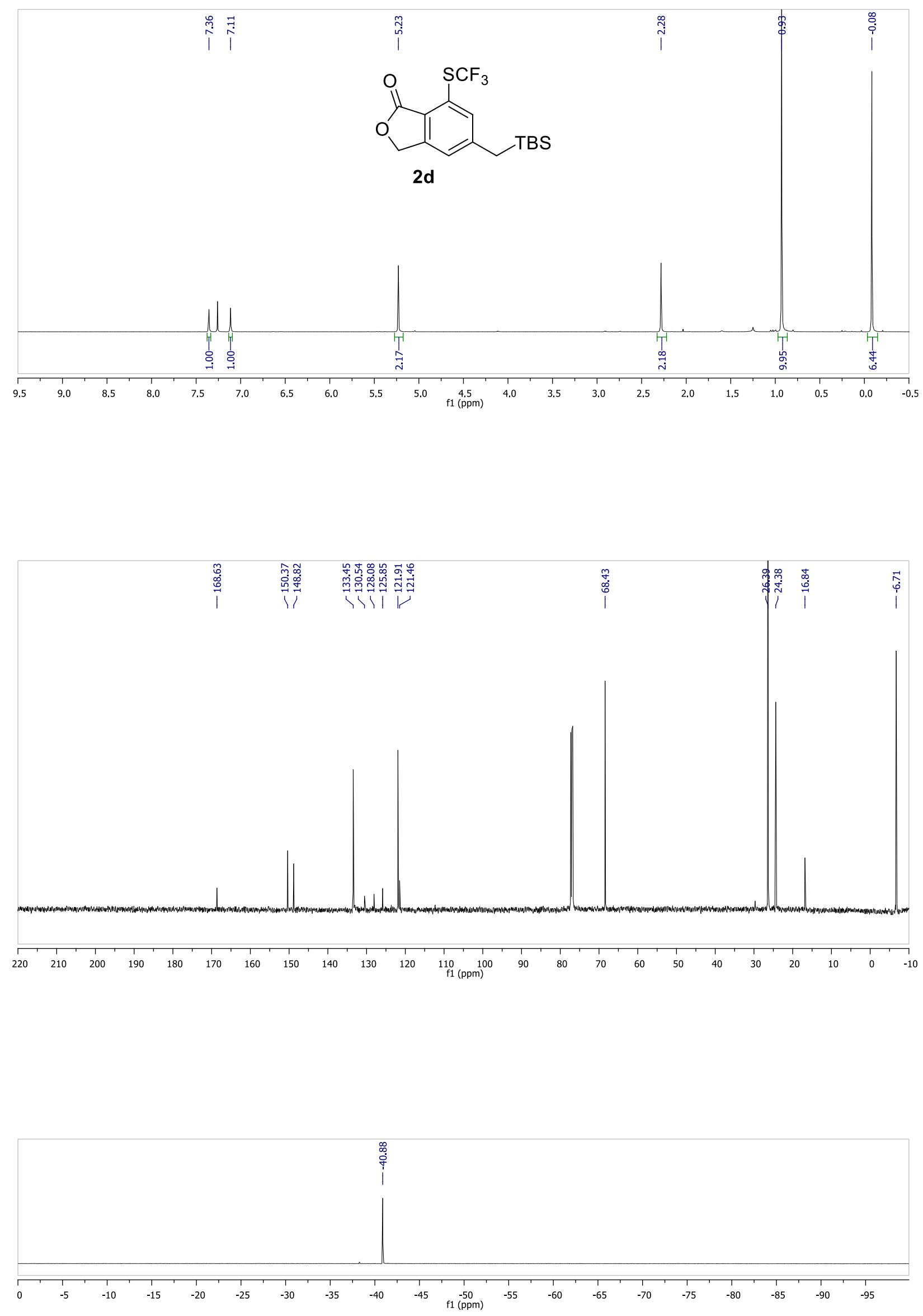

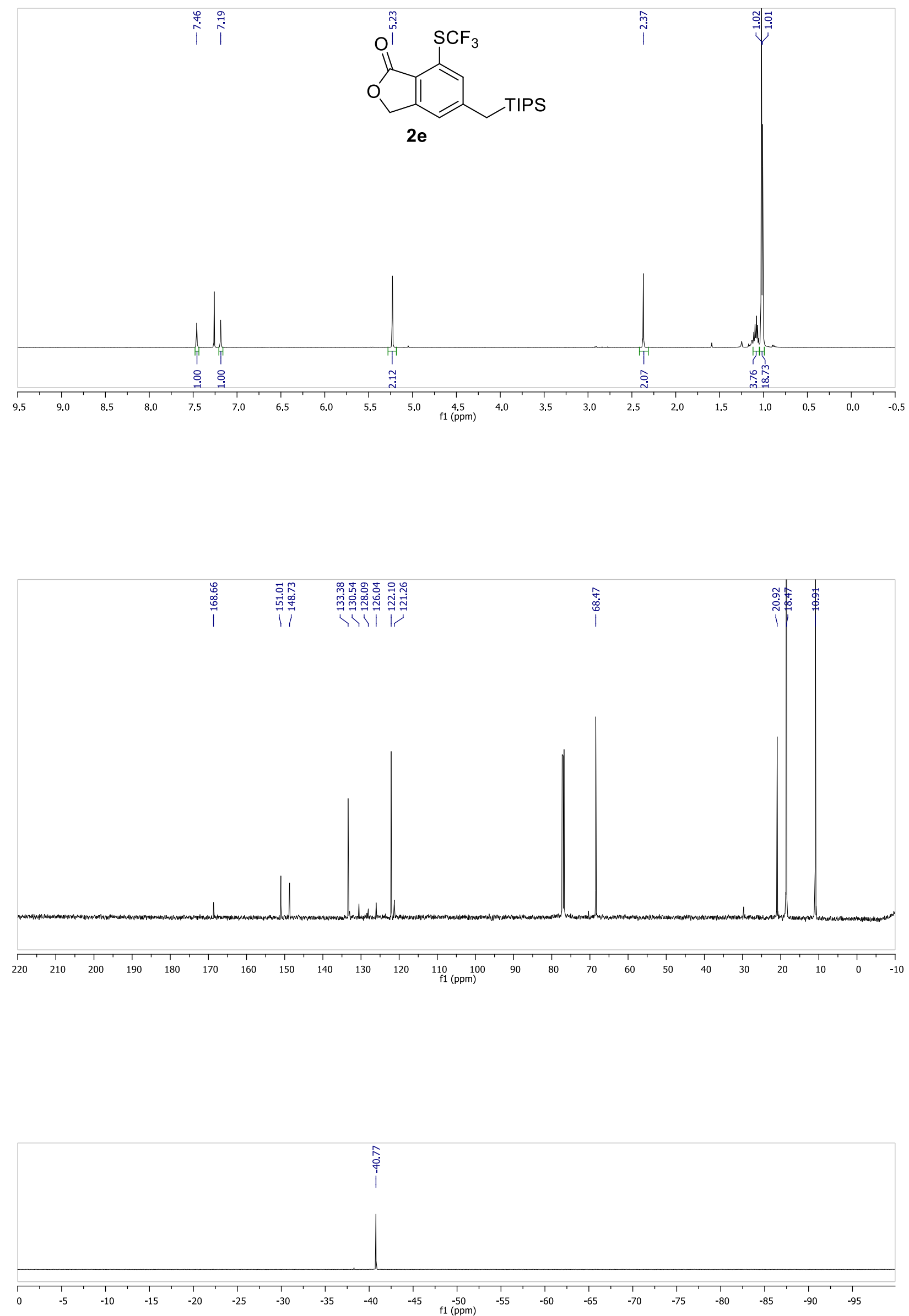

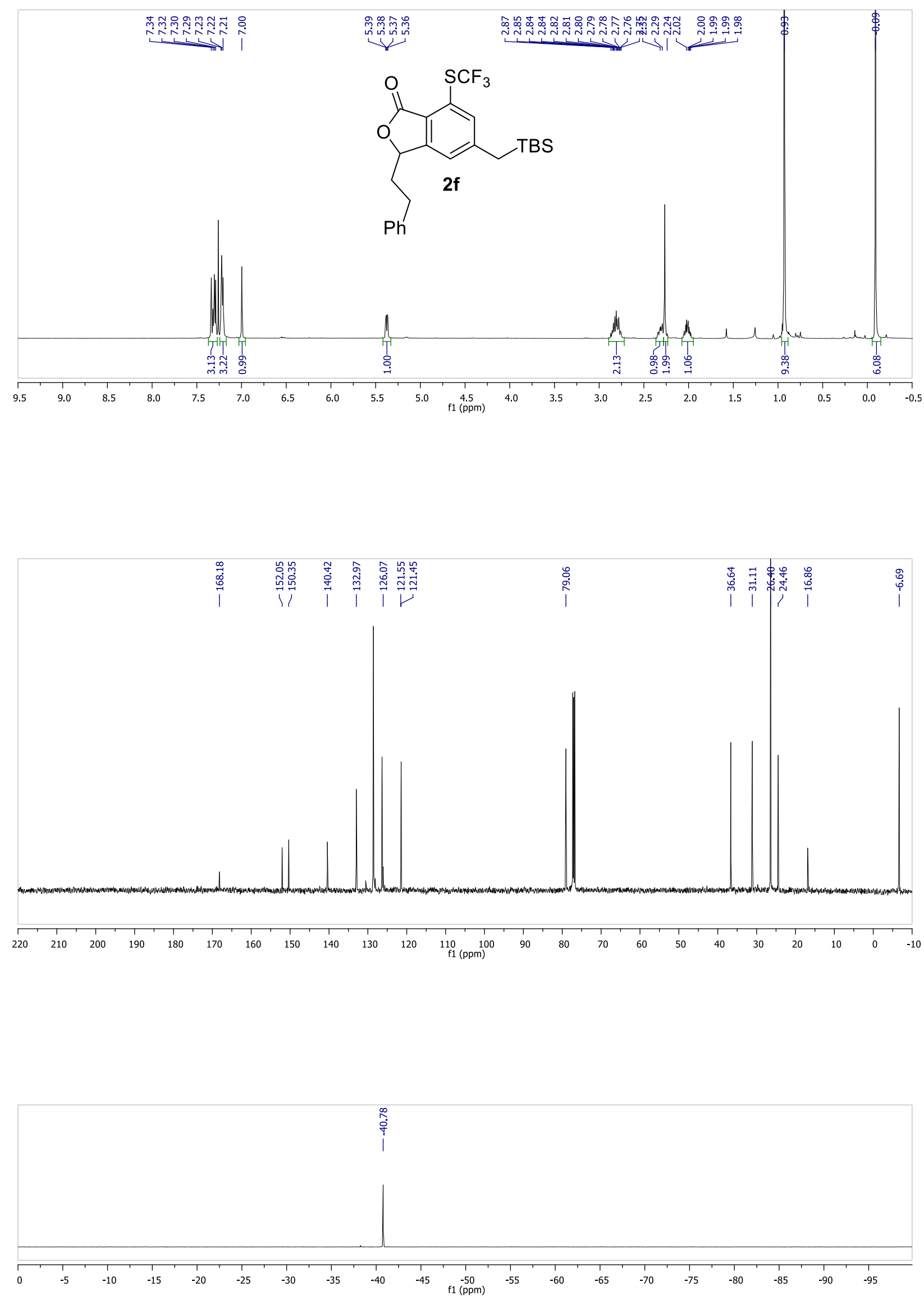

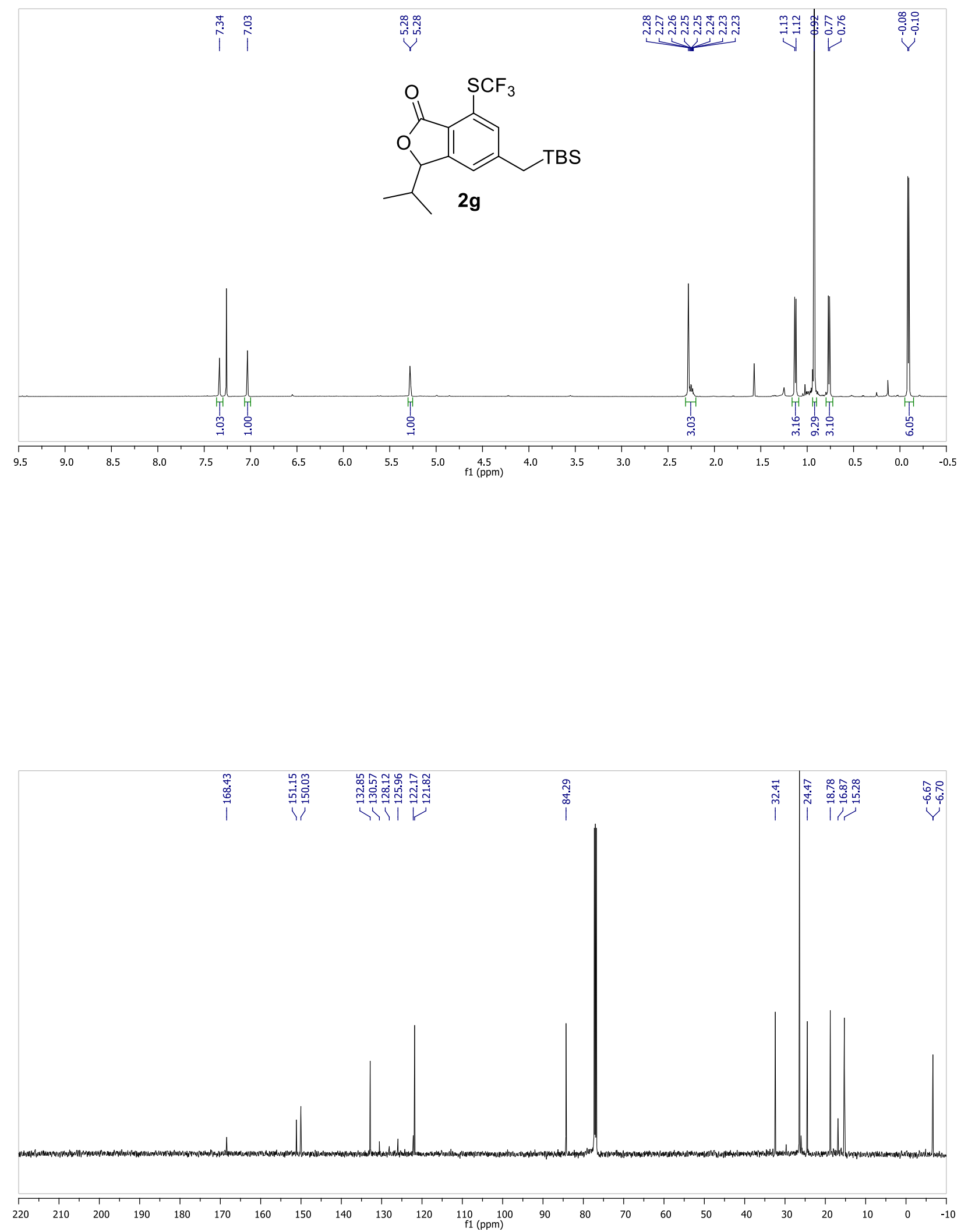

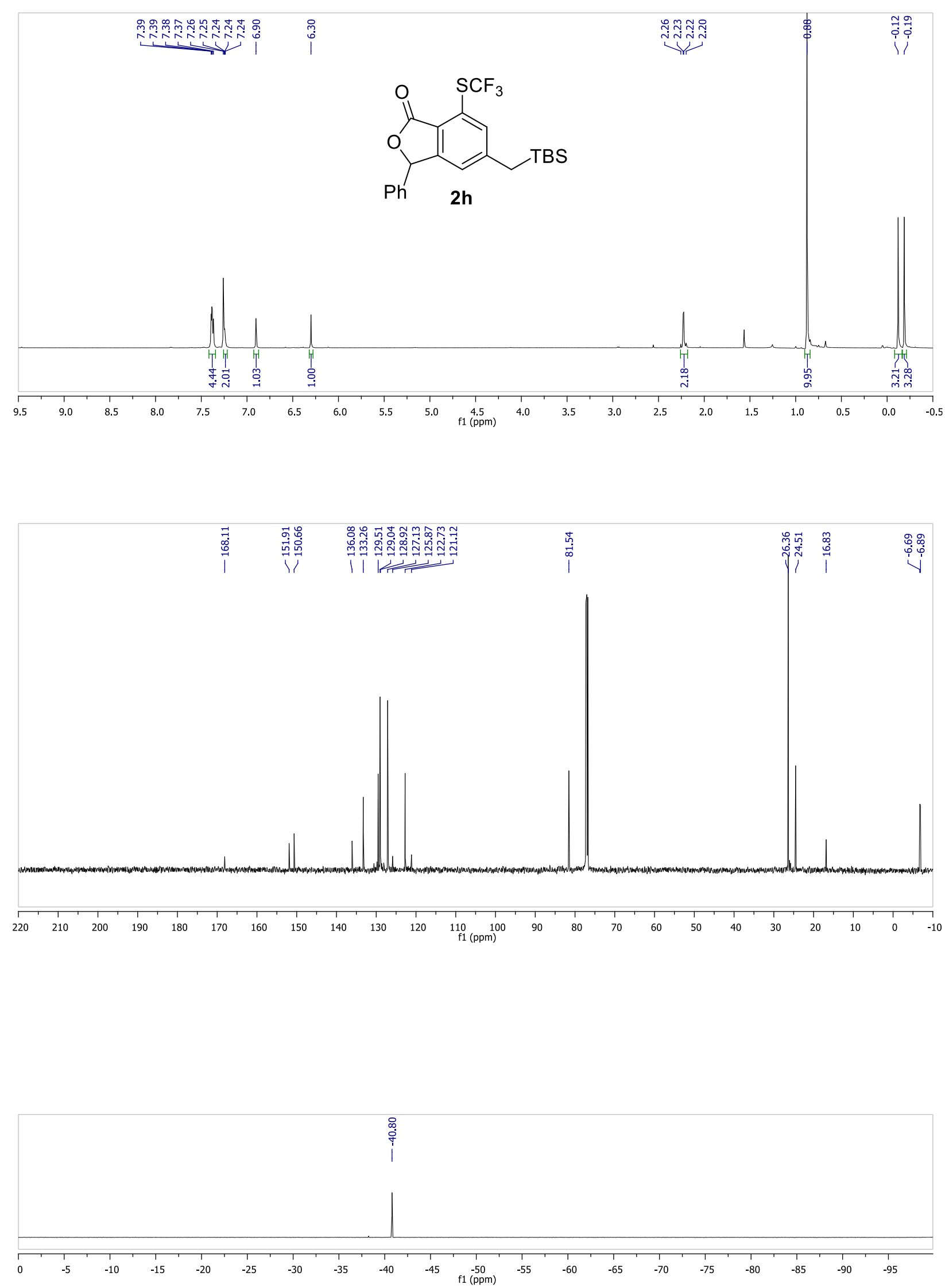

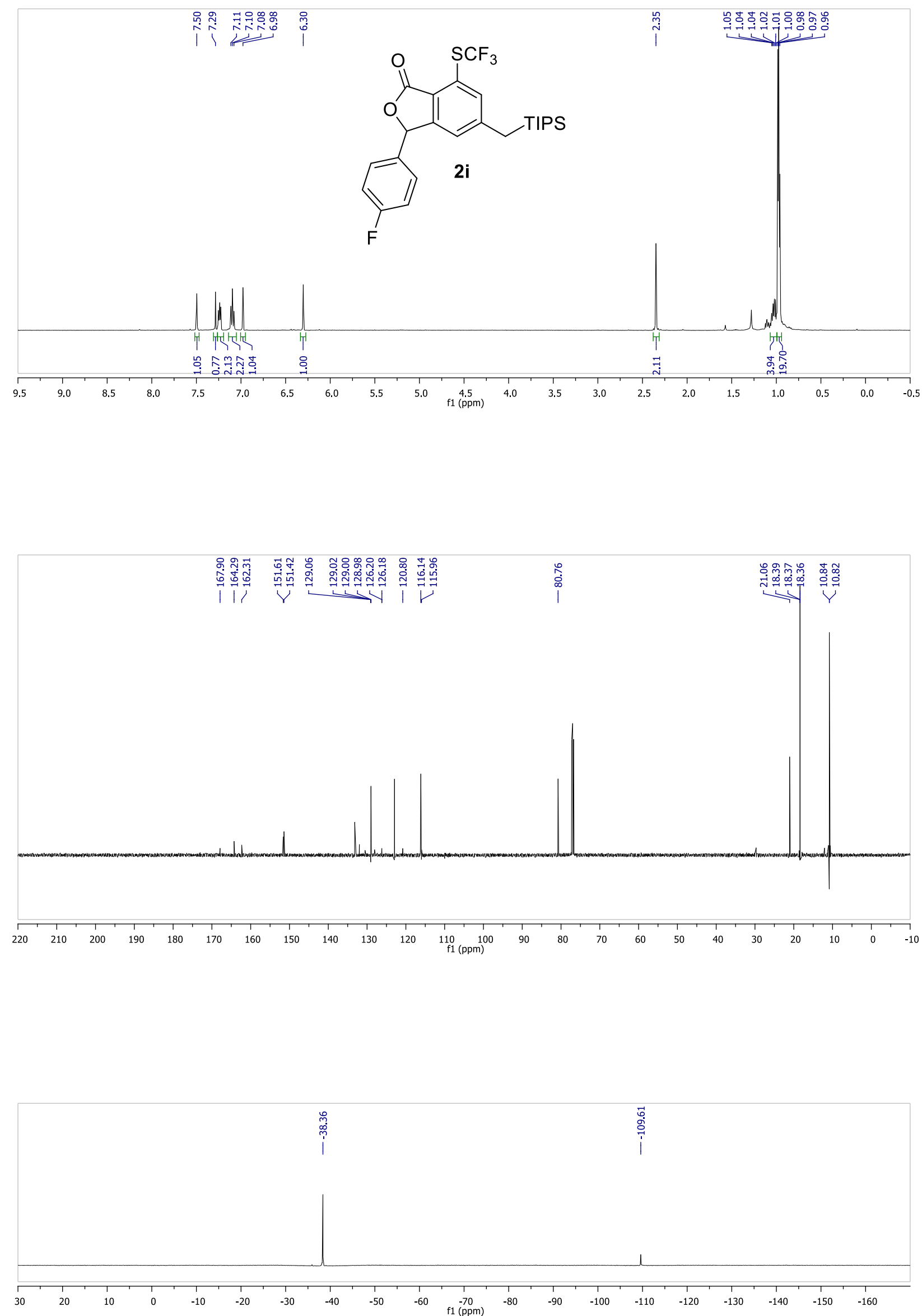

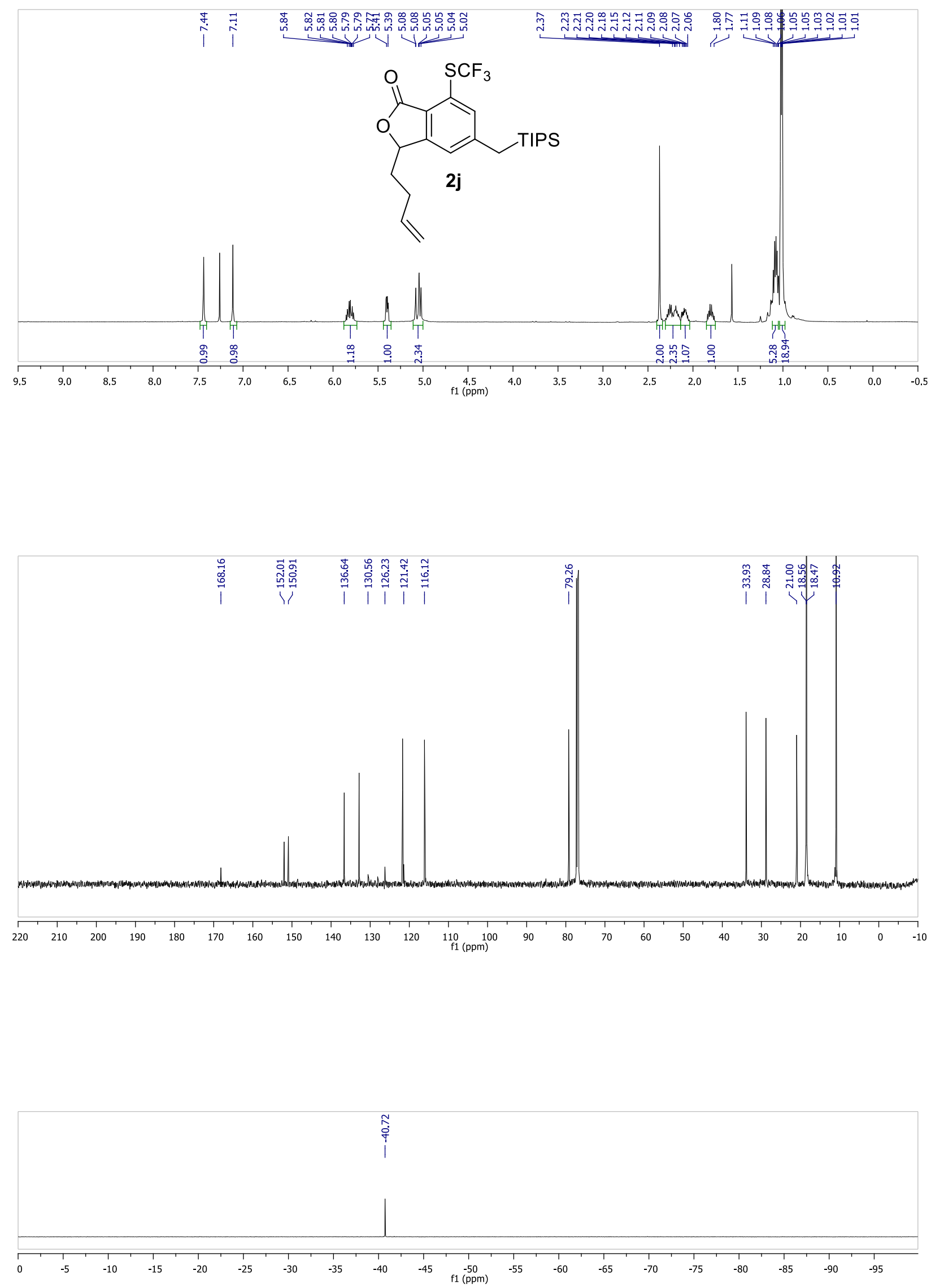

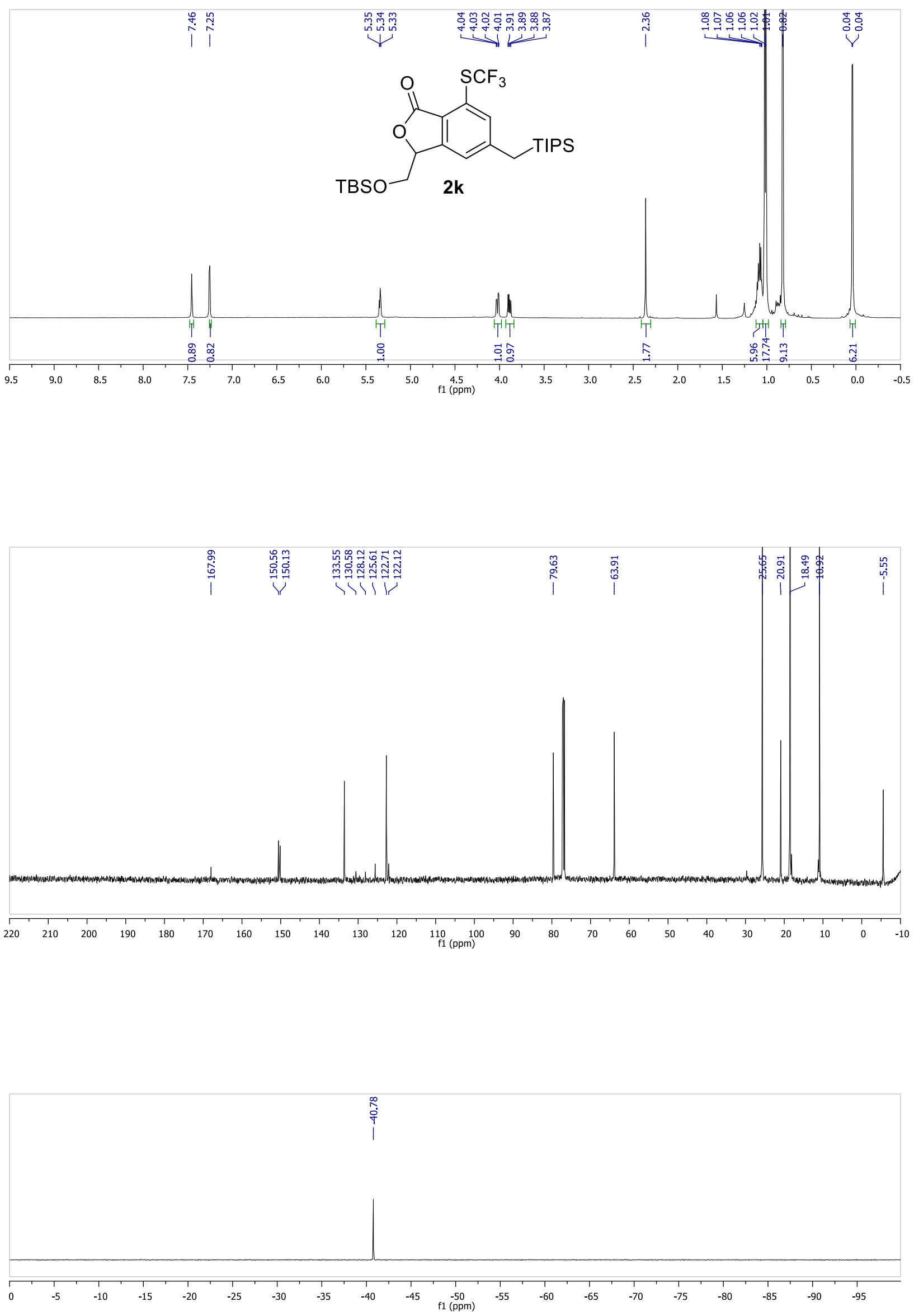

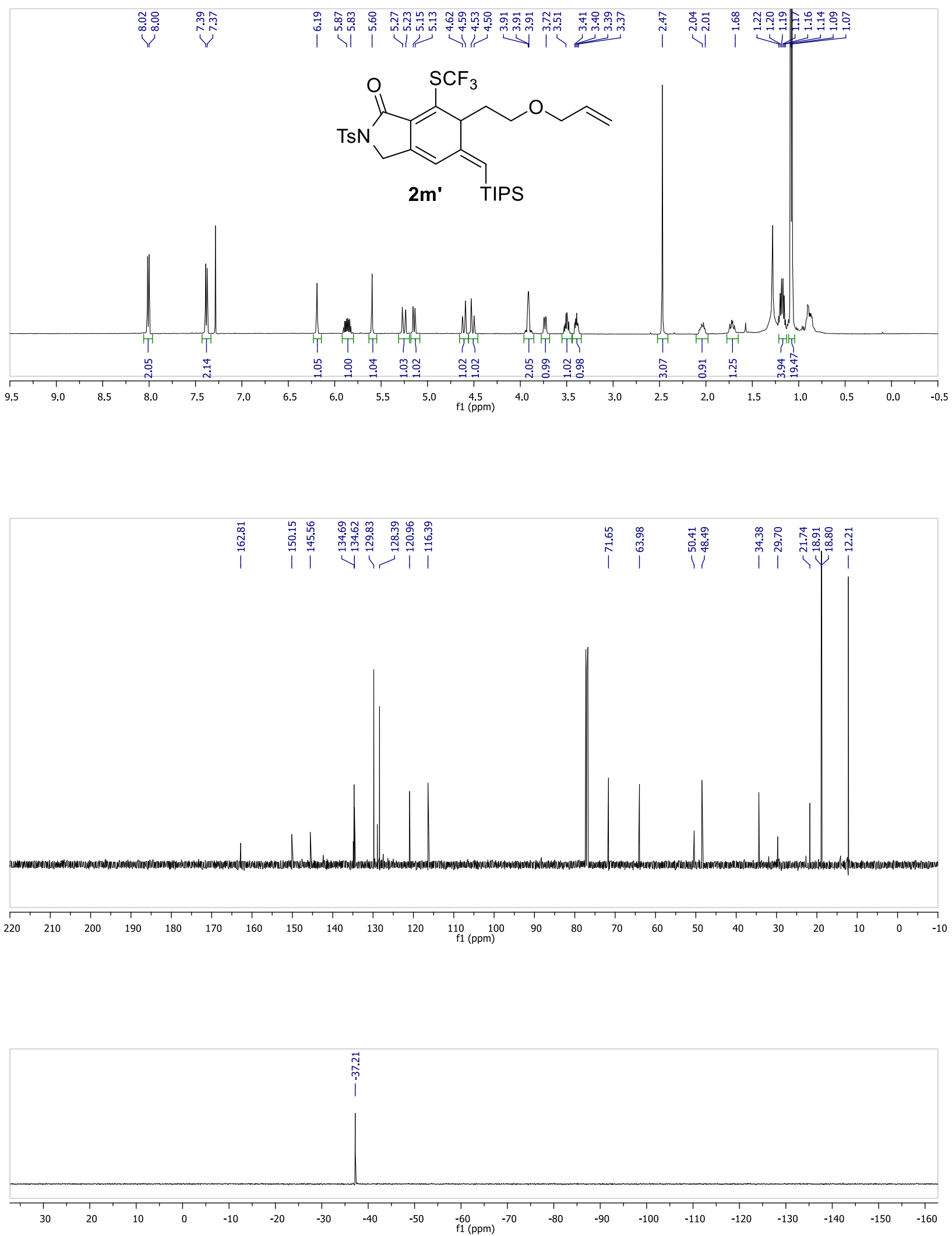

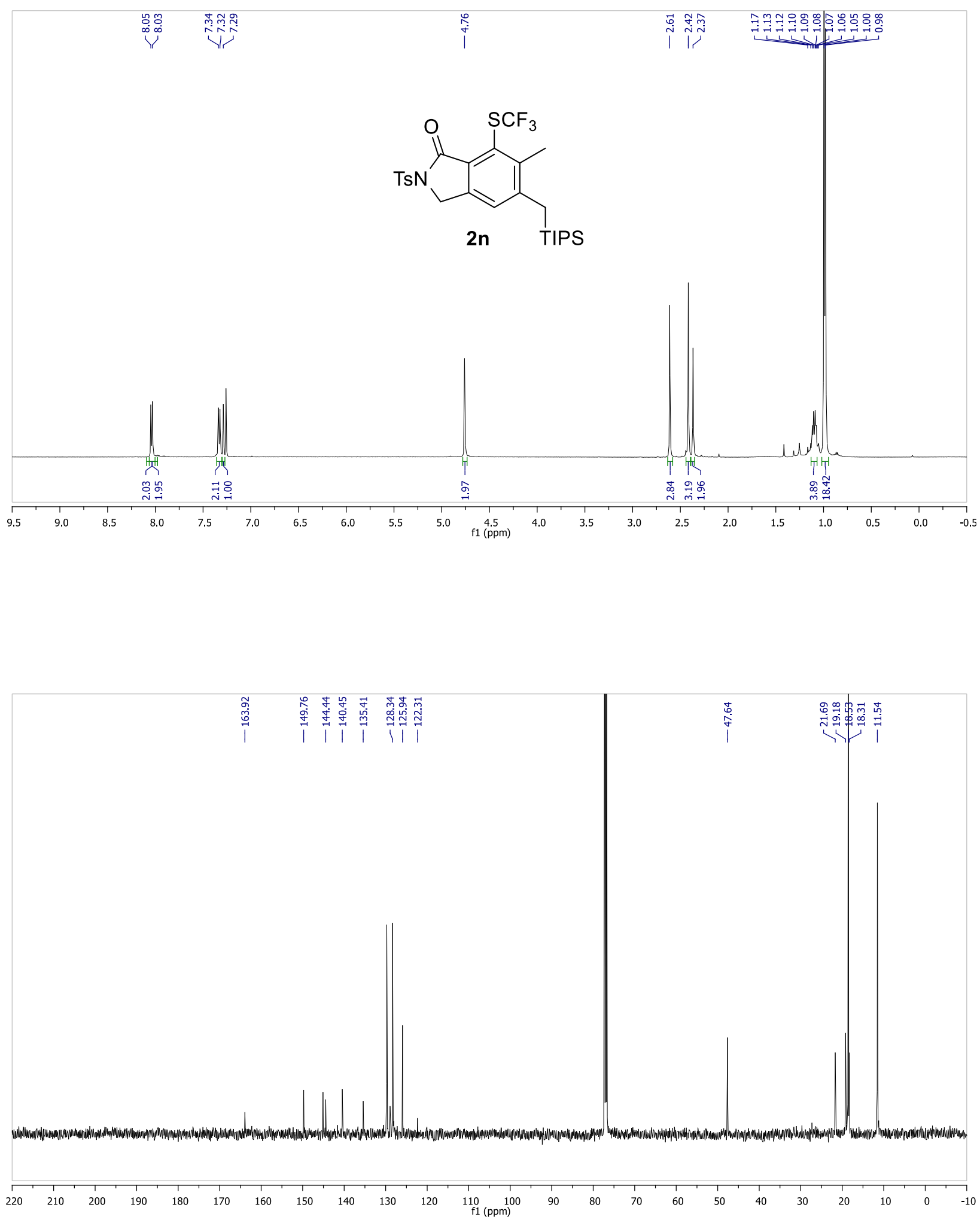

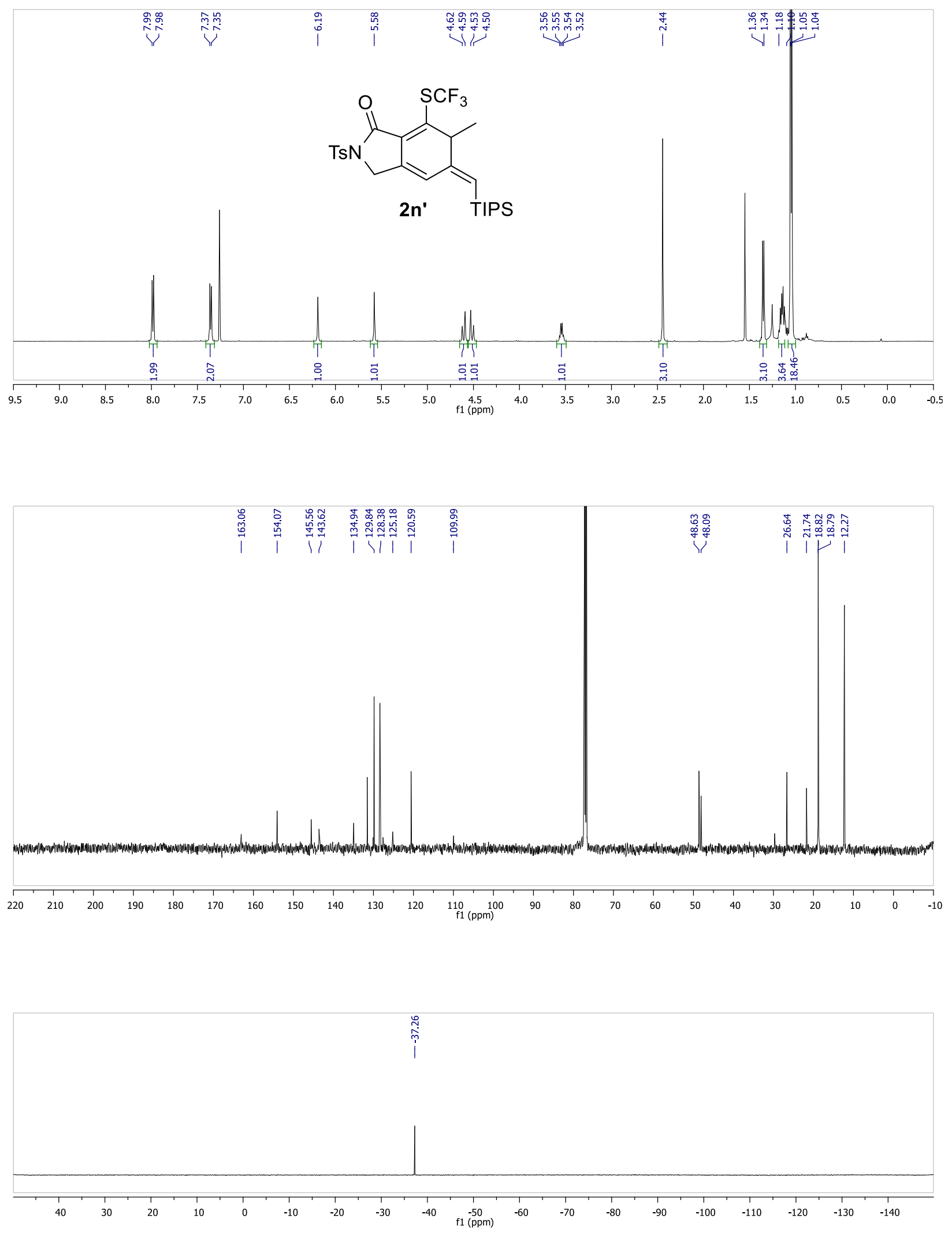

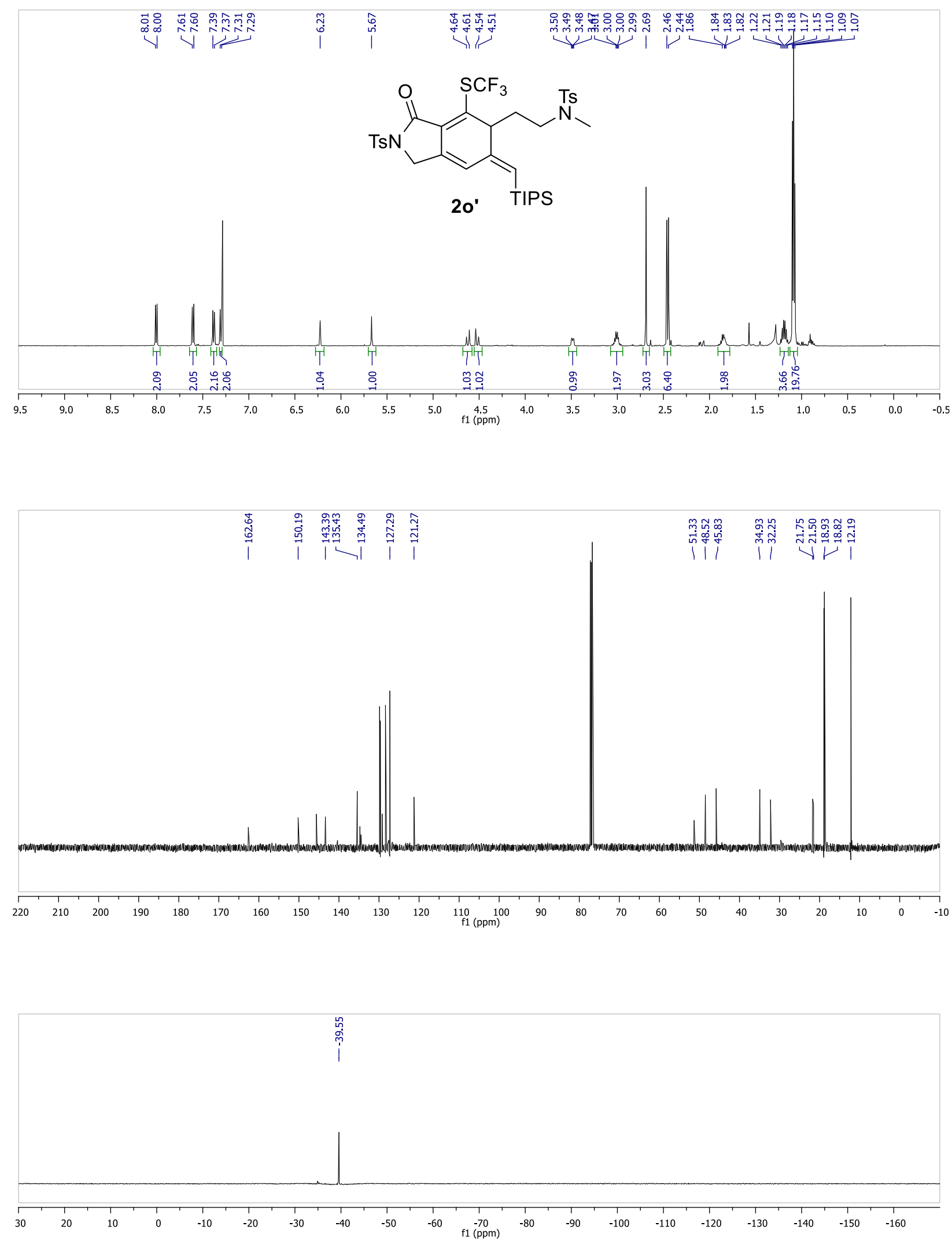

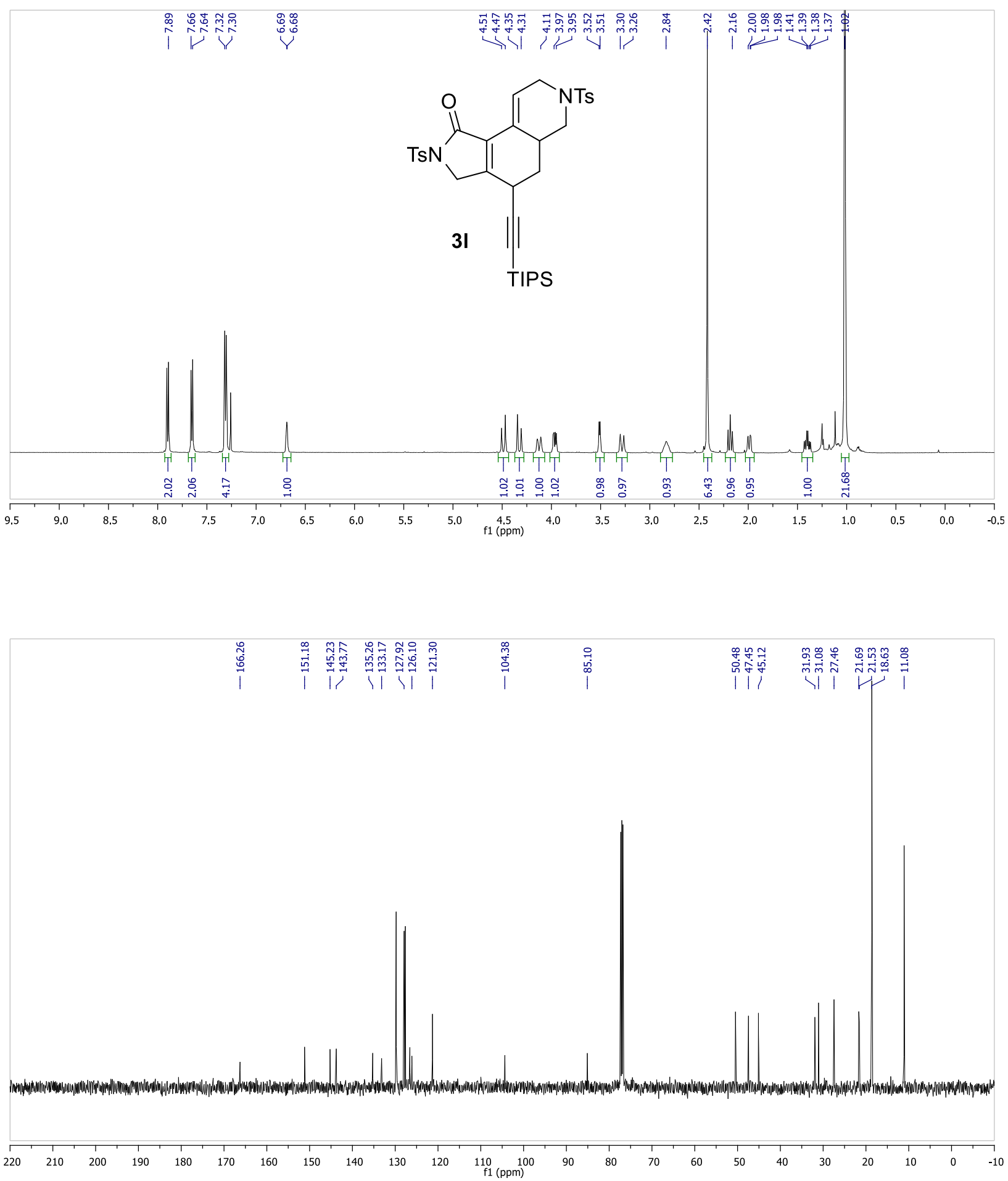


\section{Computational Details}

All calculations were carried out by using the Gaussian 09 suite of computational programs. All stationary points along the reaction coordinate were fully optimized at the DFT level using the B3LYP hybrid functional. The 6-31G(d) basis set was applied for all atoms (BSI). Frequencies were analytically computed at the same level of theory to get the thermodynamic corrections and to confirm whether the structures are minima (no imaginary frequency) or transition states (only one imaginary frequency). Intrinsic reaction coordinate (IRC) calculations were carried out to confirm that all transition state structures connect the proposed reactants and products. The solvation effect was examined by performing single-point self-consistent reaction field (SCRF) calculations based on the PCM solvation model for gas-phase optimized structures. Acetonitrile was used as the solvent, corresponding to the original experimental conditions. All SCRF calculations were done at the M06 level by using a larger basis $6-311++\mathrm{G}(\mathrm{d}, \mathrm{p})$ for all atoms (BSII). The relative free energies corrected by solvation effects from M06/BSII calculations are used for discussion.

\section{Calculated Energy Values}

Table S1. Energies (in Hartree) calculated at PCM-B3LYP/BSI// M06/ BSII

\begin{tabular}{|c|c|c|c|c|c|}
\hline Species & $\mathrm{E}_{0}^{\mathrm{a}}$ & $\mathrm{H}_{298^{\mathrm{b}}}$ & $\mathrm{G}_{298^{\mathrm{c}}}$ & $\mathrm{E}^{\mathrm{d}}$ & $\mathrm{G}_{\mathrm{Sol}}{ }^{\mathrm{e}}$ \\
\hline 1a & -2676.405342 & -2676.364352 & -2676.482083 & -2676.9664594 & -2676.42095069 \\
\hline 1n & -2715.689488 & -2715.647060 & -2715.767966 & -2716.2792305 & -2715.71480200 \\
\hline H2O & -76.385885 & -76.382106 & -76.404205 & -76.407024 & -76.4257675294 \\
\hline $\mathrm{CF}_{3} \mathrm{CH}_{2} \mathrm{OH}$ & -452.691870 & -452.685112 & -452.721493 & -452.7497195 & -452.733003142 \\
\hline$\left(\mathbf{H}_{2} \mathbf{O}\right)_{2}$ & -152.780662 & -152.773870 & -152.808359 & -152.8266189 & -152.857337450 \\
\hline TS-1a- $\mathrm{H}_{2} \mathrm{O}$ & -2752.754005 & -2752.711477 & -2752.832184 & -2753.3332143 & -2752.80217731 \\
\hline TS-1n- $\mathrm{H}_{2} \mathrm{O}$ & -2792.023007 & -2791.978962 & -2792.103605 & -2792.6306376 & -2792.08635061 \\
\hline TS-1a-CF $\mathrm{CH}_{2} \mathrm{OH}$ & -3129.059573 & -3129.012207 & -3129.146318 & -3129.6727068 & -3129.11021803 \\
\hline $\mathrm{TS}-1 \mathrm{n}-\mathrm{CF}_{3} \mathrm{CH}_{2} \mathrm{OH}$ & -3168.330788 & -3168.281901 & -3168.417495 & -3168.9722842 & -3168.39344205 \\
\hline TS-1a- $\left(\mathrm{H}_{2} \mathrm{O}\right)_{2}$ & -2829.167076 & -2829.122203 & -2829.248668 & -2829.7721663 & -2829.25206120 \\
\hline TS-1n-(H2O $)_{2}$ & -2868.440256 & -2868.393740 & -2868.524257 & -2869.0738188 & -2868.54086881 \\
\hline
\end{tabular}

${ }^{a}$ Sum of electronic and zero-point energies

${ }^{\mathrm{b}}$ Sum of electronic and thermal enthalpies

${ }^{c}$ Sum of electronic and thermal free energies

${ }^{\mathrm{d}}$ Electronic energies

e Total free energy in solution 


\section{Cartesian Coordinates for All Species}

$1 \mathbf{a}$

\begin{tabular}{|c|c|c|c|}
\hline $\mathrm{C}$ & -0.97943400 & 1.27414000 & 0.56340800 \\
\hline C & -0.36208000 & 0.05591000 & 0.83593400 \\
\hline $\mathrm{C}$ & -0.01976400 & -0.77533300 & -0.23432000 \\
\hline $\mathrm{C}$ & -0.29393800 & -0.41274400 & -1.55487200 \\
\hline $\mathrm{C}$ & -0.91535400 & 0.80965500 & -1.80218100 \\
\hline C & -1.26811700 & 1.66920500 & -0.75192000 \\
\hline $\mathrm{H}$ & -1.24420000 & 1.92992800 & 1.38901900 \\
\hline $\mathrm{H}$ & -0.13231700 & -0.24343700 & 1.85133800 \\
\hline $\mathrm{H}$ & -0.03829300 & -1.08543900 & -2.36632700 \\
\hline $\mathrm{H}$ & -1.13435000 & 1.09790500 & -2.82725400 \\
\hline C & -1.96963600 & 2.97797100 & -1.02617200 \\
\hline $\mathrm{H}$ & -3.05850600 & 2.86358300 & -0.94172000 \\
\hline $\mathrm{H}$ & -1.66870000 & 3.75100900 & -0.31129200 \\
\hline $\mathrm{H}$ & -1.75679600 & 3.34390100 & -2.03564300 \\
\hline S & 0.74737800 & -2.35651500 & 0.07981900 \\
\hline $\mathrm{O}$ & 0.26534500 & -2.87826500 & 1.35228400 \\
\hline $\mathrm{O}$ & 0.70530100 & -3.15369000 & -1.14855300 \\
\hline $\mathrm{N}$ & 2.42062100 & -1.99104800 & 0.25258300 \\
\hline $\mathrm{C}$ & 3.42585400 & -2.52147500 & -0.69180400 \\
\hline $\mathrm{C}$ & 2.96711300 & -1.27665300 & 1.32552400 \\
\hline $\mathrm{C}$ & 4.73138200 & -2.08124600 & -0.06152500 \\
\hline $\mathrm{H}$ & 3.33965700 & -3.60905100 & -0.77478200 \\
\hline C & 4.42821700 & -1.30745700 & 1.12848900 \\
\hline $\mathrm{O}$ & 2.34665100 & -0.72428900 & 2.22103200 \\
\hline C & 5.99476700 & -2.37426200 & -0.43196000 \\
\hline $\mathrm{C}$ & 7.11698500 & -1.91157200 & 0.37712500 \\
\hline $\mathrm{C}$ & 6.82479100 & -0.82663600 & 1.42575600 \\
\hline $\mathrm{C}$ & 5.38313800 & -0.72714600 & 1.89351900 \\
\hline $\mathrm{H}$ & 6.19335900 & -3.01052400 & -1.28919700 \\
\hline $\mathrm{H}$ & 3.27397100 & -2.09592200 & -1.69057200 \\
\hline S & 4.87944300 & 0.19180500 & 3.32574700 \\
\hline C & 6.46156500 & 0.82562500 & 3.98472500 \\
\hline $\mathrm{F}$ & 6.13387800 & 1.58697900 & 5.03899900 \\
\hline $\mathrm{F}$ & 7.29928300 & -0.13744600 & 4.41550800 \\
\hline $\mathrm{F}$ & 7.14661800 & 1.58929900 & 3.11377800 \\
\hline C & 8.38553500 & -2.36784300 & 0.22360100 \\
\hline $\mathrm{H}$ & 9.10811600 & -1.97001200 & 0.94275600 \\
\hline $\mathrm{Si}$ & 9.12743500 & -3.57764200 & -1.02808500 \\
\hline C & 11.03039600 & -3.53600600 & -0.75041200 \\
\hline $\mathrm{C}$ & 8.70104600 & -3.07891700 & -2.84314900 \\
\hline $\mathrm{C}$ & 8.52906300 & -5.39189400 & -0.70530000 \\
\hline $\mathrm{H}$ & 11.44636200 & -4.20314300 & -1.52169000 \\
\hline $\mathrm{H}$ & 7.68978200 & -3.47404300 & -3.02526100 \\
\hline $\mathrm{H}$ & 9.41539900 & -6.01103800 & -0.91914600 \\
\hline $\mathrm{C}$ & 8.13527300 & -5.63974500 & 0.76544500 \\
\hline C & 7.40184900 & -5.88602400 & -1.63227200 \\
\hline $\mathrm{C}$ & 11.45420300 & -4.10841600 & 0.61781400 \\
\hline $\mathrm{C}$ & 11.65811600 & -2.14343500 & -0.95805800 \\
\hline $\mathrm{C}$ & 8.63672300 & -1.56165800 & -3.10448400 \\
\hline C & 9.63562500 & -3.76715700 & -3.86013500 \\
\hline $\mathrm{H}$ & 11.49017300 & -1.75816700 & -1.96927800 \\
\hline $\mathrm{H}$ & 11.25212200 & -1.40603300 & -0.25396100 \\
\hline $\mathrm{H}$ & 12.74448100 & -2.17855300 & -0.79702700 \\
\hline $\mathrm{H}$ & 12.54771200 & -4.08931900 & 0.72410400 \\
\hline $\mathrm{H}$ & 11.04175500 & -3.52352800 & 1.45045200 \\
\hline $\mathrm{H}$ & 11.13138100 & -5.14644200 & 0.75193700 \\
\hline $\mathrm{H}$ & 7.23745100 & -5.07135100 & 1.03443800 \\
\hline $\mathrm{H}$ & 7.91563200 & -6.70293100 & 0.93423800 \\
\hline $\mathrm{H}$ & 8.92387400 & -5.35006200 & 1.46779100 \\
\hline $\mathrm{H}$ & 7.66897300 & -5.82523300 & -2.69273600 \\
\hline $\mathrm{H}$ & 7.15791200 & -6.93503400 & -1.41571900 \\
\hline $\mathrm{H}$ & 6.47785600 & -5.31146000 & -1.48823700 \\
\hline $\mathrm{H}$ & 10.66470300 & -3.39791000 & -3.77315700 \\
\hline $\mathrm{H}$ & 9.66608600 & -4.85619800 & -3.73159900 \\
\hline $\mathrm{H}$ & 9.30694000 & -3.56846800 & -4.88936400 \\
\hline $\mathrm{H}$ & 7.92007700 & -1.06268500 & -2.44390900 \\
\hline $\mathrm{H}$ & 9.60915200 & -1.07862400 & -2.95571300 \\
\hline $\mathrm{H}$ & 8.33199700 & -1.36106100 & -4.14110600 \\
\hline $\mathrm{H}$ & 7.08311900 & 0.15283500 & 0.99333200 \\
\hline $\mathrm{H}$ & 7.49862000 & -0.95974200 & 2.27402000 \\
\hline
\end{tabular}

1n

$\begin{array}{ll}\mathrm{H} & \\ \mathrm{H} & -0 . \\ \mathrm{C} & -1.93 \\ \mathrm{H} & -2.13 \\ \mathrm{H} & -2.8 \\ \mathrm{H} & -1.38 \\ \mathrm{~S} & 1.26 \\ \mathrm{O} & 0.60 \\ \mathrm{O} & 1.63 \\ \mathrm{~N} & 2.77 \\ \mathrm{C} & 4.04\end{array}$

$\begin{array}{lll}0.89278400 & -5.71702500 & -1.67335700\end{array}$

$\begin{array}{lll}-0.42240600 & -4.90420700 & -3.62091300\end{array}$

$\begin{array}{lll}-1.93607300 & -2.63228800 & -3.80244000\end{array}$

$\begin{array}{lll}-2.13497900 & -3.42974200 & -4.52516600\end{array}$

$\begin{array}{lll}-2.89590400 & -2.20439700 & -3.49348100\end{array}$

$\begin{array}{lll}-1.38360700 & -1.84176900 & -4.32741100\end{array}$

$\begin{array}{lll}1.26511900 & -4.65689200 & 0.96331500\end{array}$

$\begin{array}{lll}0.60397900 & -4.24875100 & 2.19710300\end{array}$

$\begin{array}{lll}1.63573300 & -6.05234200 & 0.71382500\end{array}$

$\begin{array}{lll}2.77028000 & -3.82822400 & 0.85362600\end{array}$

$\begin{array}{lll}4.04447900 & -4.56683700 & 0.73664200\end{array}$

$\begin{array}{lll}2.92487400 & -2.44827500 & 1.03567600\end{array}$

$\begin{array}{lll}5.06909800 & -3.45190900 & 0.76352900\end{array}$

$\begin{array}{lll}4.15850100 & -5.26611000 & 1.57247900\end{array}$

$\begin{array}{lll}4.37869100 & -2.19548300 & 0.98788600\end{array}$

$\begin{array}{lll}2.02733200 & -1.63561000 & 1.19323700\end{array}$

$\begin{array}{lll}6.39357700 & -3.50777700 & 0.50834600\end{array}$

$\begin{array}{lll}7.17612500 & -2.27648500 & 0.49535900\end{array}$

$\begin{array}{lll}6.55088200 & -1.05402800 & 1.18978700\end{array}$

$\begin{array}{lll}5.02958300 & -1.01032000 & 1.09041300\end{array}$

$\begin{array}{lll}6.87316100 & -4.44354300 & 0.23726600\end{array}$

$\begin{array}{lrr}4.07116000 & -5.14713100 & -0.19115300\end{array}$

$\begin{array}{lrr}4.06674000 & 0.46918500 & 1.29334400\end{array}$

$\begin{array}{lll}5.27656500 & 1.79903400 & 0.96762300\end{array}$

$\begin{array}{lll}4.57147700 & 2.94016200 & 0.98323300\end{array}$

$\begin{array}{lll}6.25105300 & 1.90773900 & 1.89200600\end{array}$

$\begin{array}{lll}5.87932200 & 1.69209900 & -0.23081200\end{array}$

$\begin{array}{lll}6.94285900 & -1.07246500 & 2.69495100\end{array}$

$\begin{array}{lll}8.03457200 & -1.07059500 & 2.77879800\end{array}$

$\begin{array}{lll}6.54763000 & -0.19698500 & 3.21608900\end{array}$

$\begin{array}{rrr}6.55958800 & -1.97436900 & 3.18203900\end{array}$

$\begin{array}{rrr}8.42256100 & -2.18326100 & -0.03404100\end{array}$

$\begin{array}{lll}8.85533400 & -1.17838900 & -0.00653000\end{array}$

$\begin{array}{lll}9.56371400 & -3.48502100 & -0.80012200\end{array}$

$\begin{array}{lll}11.00496200 & -2.51586200 & -1.62964300\end{array}$

$\begin{array}{lll}8.64947500 & -4.57649500 & -2.10028600\end{array}$

$\begin{array}{lll}10.33041600 & -4.62753500 & 0.56445800\end{array}$

$\begin{array}{lll}11.62140600 & -3.28641300 & -2.11853300\end{array}$

$\begin{array}{lll}8.09745700 & -5.32332300 & -1.50964000\end{array}$

$\begin{array}{lll}11.34984400 & -4.84118100 & 0.20447400\end{array}$

$\begin{array}{lll}10.46004800 & -3.92484000 & 1.93184600\end{array}$

$\begin{array}{lll}9.62508800 & -5.98539800 & 0.75194100\end{array}$

$\begin{array}{lll}11.91543200 & -1.78519000 & -0.62258000\end{array}$

$\begin{array}{lll}10.53640700 & -1.53647200 & -2.72410200\end{array}$

$\begin{array}{lll}7.61036400 & -3.83729400 & -2.96571300\end{array}$

$\begin{array}{lll}9.63882400 & -5.35794400 & -2.99069600\end{array}$

$\begin{array}{lll}10.00260000 & -2.04349000 & -3.53462900\end{array}$

$\begin{array}{lll}9.86563600 & -0.76734700 & -2.32041400\end{array}$

$\begin{array}{lll}11.39323200 & -1.01482000 & -3.17241600\end{array}$

$\begin{array}{lll}12.73267200 & -1.26851400 & -1.14457900\end{array}$

$\begin{array}{lll}11.36761700 & -1.02197300 & -0.05435900\end{array}$

$\begin{array}{lll}12.37250400 & -2.47083800 & 0.09890000\end{array}$

$\begin{array}{lll}9.47329700 & -3.71678400 & 2.36212500\end{array}$

$\begin{array}{lll}11.00271300 & -4.56051700 & 2.64507100\end{array}$

$\begin{array}{lll}10.99377500 & -2.97084300 & 1.86838600\end{array}$

$\begin{array}{lll}9.59260600 & -6.57571600 & -0.16996200\end{array}$

$\begin{array}{lll}10.14640600 & -6.58837400 & 1.50796800\end{array}$

$\begin{array}{lll}8.59264600 & -5.86161400 & 1.10470200\end{array}$

$\begin{array}{lll}10.20904200 & -4.68577200 & -3.64310700\end{array}$

$\begin{array}{lll}10.36182800 & -5.93899700 & -2.40512800\end{array}$

$\begin{array}{lll}9.10371200 & -6.06225200 & -3.64214600\end{array}$

$\begin{array}{lll}6.85698800 & -3.32767500 & -2.35658200\end{array}$

$\begin{array}{lll}8.07685400 & -3.08252000 & -3.60856600\end{array}$

$\begin{array}{lll}7.08696700 & -4.54322100 & -3.62560900\end{array}$

$\begin{array}{rrr}6.98623000 & -0.16126100 & 0.74022800\end{array}$

H2O

$1.20645000 \quad 0.70316800$

$2.17386300 \quad 0.75067200$

0.00000000

0.00000000

$\begin{array}{lll}0.92829700 & 1.63095300 \quad 0.00000000\end{array}$

\section{$\mathrm{CF}_{3} \mathrm{CH}_{2} \mathrm{OH}$}

$\begin{array}{rrr}-1.13795700 & -2.42417200 & -1.40992100 \\ -0.40225000 & -2.86511400 & -0.31316100 \\ 0.33515400 & -4.04642000 & -0.43281300 \\ 0.33539600 & -4.78842300 & -1.61560600 \\ -0.41043700 & -4.32812500 & -2.69915300 \\ -1.15187300 & -3.14108000 & -2.61651600 \\ -1.71560300 & -1.50701900 & -1.32612800 \\ -0.38535300 & -2.30632400 & 0.61442400\end{array}$

$\mathrm{C}$
$\mathrm{C}$
$\mathrm{H}$
$\mathrm{H}$
$\mathrm{F}$
$\mathrm{F}$
$\mathrm{F}$
$\mathrm{O}$




\section{TS-1n- $\mathrm{H}_{2} \mathrm{O}$}

\section{$(\mathrm{H} 2 \mathrm{O})_{2}$}

$\mathrm{O}$
$\mathrm{H}$
$\mathrm{H}$
$\mathrm{O}$
$\mathrm{H}$
$\mathrm{H}$

\section{TS-1a-H2O}

C

$\begin{array}{ccc}0.12255300 & 0.48490000 & 0.46001100 \\ 0.93265200 & 0.86736200 & 0.07023200 \\ -0.59418800 & 0.85304400 & -0.07617100 \\ 2.73426400 & 1.30515500 & -0.37799000 \\ 3.04888600 & 0.39134000 & -0.46661400 \\ 3.02727300 & 1.57208400 & 0.50784900\end{array}$

$\begin{array}{lll}-0.85676900 & 0.67364100 & -2.07110500\end{array}$ $\begin{array}{lll}-0.40690700 & -0.59825700 & -1.72756900\end{array}$

$\begin{array}{lll}0.27936400 & -1.34794700 & -2.68707700\end{array}$

$\begin{array}{lll}0.50542000 & -0.85257700 & -3.97252200\end{array}$

$\begin{array}{lll}0.04290000 & 0.42244500 & -4.29408200\end{array}$

$\begin{array}{lll}-0.63886200 & 1.20499100 & -3.35188900\end{array}$

$\begin{array}{lll}-1.39236800 & 1.26346000 & -1.33137600\end{array}$

$\begin{array}{lll}-0.56892600 & -1.00359200 & -0.73642500\end{array}$

$\begin{array}{lll}1.01445600 & -1.46582300 & -4.70814800\end{array}$

$\begin{array}{rrr}0.20756200 & 0.81163700 & -5.29564000 \\ -1.11662200 & 2.59519000 & -3.69759300\end{array}$

$\begin{array}{lll}-2.10659300 & 2.79473400 & -3.27304900\end{array}$

$\begin{array}{lll}-0.43372400 & 3.35580300 & -3.29661300\end{array}$

$\begin{array}{lll}-1.17406300 & 2.74271700 & -4.78054500\end{array}$

$\begin{array}{lll}0.83142900 & -3.00008400 & -2.29450800\end{array}$

$\begin{array}{lll}-0.09812500 & -3.59795600 & -1.34278000\end{array}$

$\begin{array}{lll}1.20384000 & -3.68250600 & -3.53713400\end{array}$

$\begin{array}{lll}2.34069700 & -2.76788400 & -1.50956600\end{array}$

$\begin{array}{lll}3.57139800 & -3.40899900 & -2.00873600\end{array}$

$\begin{array}{lll}2.49882100 & -2.17188800 & -0.24507800\end{array}$

$\begin{array}{lll}4.56514900 & -3.11209100 & -0.90629200\end{array}$

$\begin{array}{lll}3.42191400 & -4.48394800 & -2.15413800\end{array}$

$\begin{array}{lll}3.90611000 & -2.37276700 & 0.10906600\end{array}$

$\begin{array}{rrr}1.62892400 & -1.60234000 & 0.39756400\end{array}$

$\begin{array}{lll}5.88390800 & -3.47478100 & -0.83215900 \\ 6.5286900 & -3.11828200 & 0.32390700\end{array}$

$\begin{array}{lll}6.65286900 & -3.11828200 & 0.32390700 \\ 5.97945600 & -2.28605500 & 1.33885300\end{array}$

$\begin{array}{lll}4.58521800 & -1.98207500 & 1.25108500\end{array}$

$\begin{array}{lll}6.34468000 & -4.05835200 & -1.62143000\end{array}$

$\begin{array}{lll}3.87075100 & -2.97944000 & -2.97093100\end{array}$

$\begin{array}{lll}3.65605100 & -1.09036900 & 2.48135100\end{array}$

$\begin{array}{lll}4.94996000 & -0.60009200 & 3.67001400\end{array}$

$\begin{array}{lll}4.34258600 & 0.17106800 & 4.58195400\end{array}$

$\begin{array}{lll}5.52686500 & -1.63027900 & 4.31904300\end{array}$

$\begin{array}{lll}5.94980700 & 0.12257600 & 3.11606200\end{array}$

$\begin{array}{lll}8.05413300 & -3.28472000 & 0.44788700\end{array}$

$\begin{array}{lll}8.39279200 & -3.20034500 & 1.48966100\end{array}$

$\begin{array}{lll}9.28036000 & -4.26248200 & -0.58782600\end{array}$

$\begin{array}{lll}10.99144300 & -3.78214100 & 0.16862800\end{array}$

$\begin{array}{lll}9.11895000 & -3.87475300 & -2.47123500\end{array}$

$\begin{array}{lll}9.06662400 & -6.16415700 & -0.34037800\end{array}$

$\begin{array}{rrr}10.82436800 & -3.89746100 & 1.25283800\end{array}$

$\begin{array}{lll}8.19320200 & -4.38234000 & -2.78589900\end{array}$

$\begin{array}{lll}9.90646900 & -6.64256700 & -0.86876100\end{array}$

$\begin{array}{lll}9.16651000 & -6.55885200 & 1.14699700\end{array}$

$\begin{array}{lll}7.76553800 & -6.72831700 & -0.94221700\end{array}$

$\begin{array}{lll}11.39851700 & -2.31413200 & -0.06653300\end{array}$

$\begin{array}{lll}12.15975700 & -4.71744400 & -0.20125900\end{array}$

$\begin{array}{lll}8.94232700 & -2.38112400 & -2.81037500\end{array}$

$\begin{array}{lll}10.25620700 & -4.48627100 & -3.31501400\end{array}$

$\begin{array}{llr}11.93690200 & -5.76704300 & 0.01811400\end{array}$

$\begin{array}{lll}12.41235400 & -4.65104800 & -1.26583800\end{array}$

$\begin{array}{lll}13.06522000 & -4.45012900 & 0.36133000\end{array}$

$\begin{array}{lll}12.29028600 & -2.05367600 & 0.52073500\end{array}$

$\begin{array}{lll}11.64341900 & -2.12961100 & -1.11929300\end{array}$

$\begin{array}{lll}10.60618000 & -1.60773300 & 0.21014700\end{array}$

$\begin{array}{lll}8.36074400 & -6.09698800 & 1.73125400\end{array}$

$\begin{array}{lll}9.07765400 & -7.64688600 & 1.27209900\end{array}$

$\begin{array}{lll}10.11804700 & -6.25512600 & 1.59894000\end{array}$

$\begin{array}{lll}7.69069500 & -6.54750500 & -2.02092100\end{array}$

$\begin{array}{lll}7.70461300 & -7.81506000 & -0.79124800 \\ 6.88068200 & -6.28823600 & -0.46585400\end{array}$

$\begin{array}{lll}11.21392600 & -3.99159600 & -3.11420400\end{array}$

$\begin{array}{lll}11.21392600 & -3.99159600 & -3.11420400 \\ 10.39107000 & -5.55719600 & -3.12252000\end{array}$

$\begin{array}{lll}10.05268300 & -4.36879700 & -4.38834800\end{array}$

$\begin{array}{lll}8.09757200 & -1.93116900 & -2.27720200\end{array}$

$\begin{array}{lll}9.83674500 & -1.79899100 & -2.55893300\end{array}$

$\begin{array}{lll}8.76374700 & -2.24585200 & -3.88640100\end{array}$

$\begin{array}{lll}6.76242500 & -1.13074000 & 0.91142700\end{array}$

$\begin{array}{lll}6.41813100 & -2.32616400 & 2.33337800\end{array}$

$\begin{array}{lll}7.77355100 & -0.61269900 & 0.48133200\end{array}$

$\begin{array}{lll}8.16363100 & -1.64253100 & 0.29586500\end{array}$

$\begin{array}{lll}8.25048600 & -0.24860700 & 1.24877600\end{array}$
$-0.00795200$

0.21692700
0.58189400

2.36250600

$1.00038300-1.86979500$

$\begin{array}{ll}0.54346500 & 0.42044800\end{array}$

$\begin{array}{lll}0.71199800 & 1.41963400 & -0.65853900\end{array}$

$\begin{array}{lll}0.47945600 & 2.77890100 & -0.45477100\end{array}$

$\begin{array}{llr}0.11644200 & 3.27052700 & 0.80664100\end{array}$

$\begin{array}{lll}-0.28716800 & 2.72745000 & 2.85512600\end{array}$

$\begin{array}{lll}0.13138000 & 0.30207700 & 2.51318100\end{array}$

$\begin{array}{lll}0.97410200 & 1.03693700 & -1.63889800\end{array}$

$\begin{array}{lll}0.57579500 & 3.46713900 & -1.29074600\end{array}$

$\begin{array}{lll}-0.16129500 & 4.74007400 & 1.01646400\end{array}$

$\begin{array}{lll}0.25632200 & 5.34764000 & 0.20754900\end{array}$

$\begin{array}{lll}-1.24136000 & 4.93430000 & 1.05130100\end{array}$

$\begin{array}{lll}0.26076700 & 5.09532000 & 1.96305700\end{array}$

$\begin{array}{lll}0.84255200 & -1.20187600 & 0.14401900\end{array}$

$\begin{array}{lll}-0.02279000 & -1.96034400 & 1.03956200\end{array}$

$\begin{array}{lll}0.85862800 & -1.45122700 & -1.30080500\end{array}$

$\begin{array}{lll}2.47579400 & -1.46939400 & 0.60070600\end{array}$

$\begin{array}{lll}3.45054300 & -2.01516300 & -0.35237300\end{array}$

$\begin{array}{lll}2.95514500 & -1.36764800 & 1.92434300\end{array}$

$\begin{array}{lll}4.66472200 & -2.21760700 & 0.52190600\end{array}$

$\begin{array}{lll}3.09312500 & -2.95355900 & -0.79023400\end{array}$

$\begin{array}{lll}4.36106200 & -1.85084200 & 1.86087300\end{array}$

$\begin{array}{lll}2.30681800 & -0.94567600 & 2.86169000\end{array}$

$\begin{array}{lll}5.89709200 & -2.66822200 & 0.14351600\end{array}$

$\begin{array}{lll}6.95845100 & -2.80760700 & 1.09877600\end{array}$

$\begin{array}{lll}6.65534800 & -2.43049400 & 2.50625400\end{array}$

$\begin{array}{lll}5.31914600 & -2.00225600 & 2.84630100\end{array}$

$\begin{array}{lll}6.08353500 & -2.94789900 & -0.88863400\end{array}$

$\begin{array}{lll}3.63445900 & -1.31292100 & -1.17290300\end{array}$

$\begin{array}{lll}4.93984000 & -1.48655800 & 4.53562800\end{array}$

$\begin{array}{lll}4.13980700 & -3.00698600 & 5.15518800\end{array}$

$\begin{array}{lll}3.72241000 & -2.74827700 & 6.40433200\end{array}$

$3.09307200 \quad-3.39491500 \quad 4.42072300$

$\begin{array}{lll}4.99223600 & -4.05215800 & 5.20929700\end{array}$

$\begin{array}{lll}8.29638100 & -3.04757400 & 0.71010300\end{array}$

$\begin{array}{lll}8.94287300 & -3.38619500 & 1.52329200\end{array}$

$\begin{array}{lll}8.98674000 & -3.52866400 & -0.97002500\end{array}$

$\begin{array}{lll}10.90478500 & -3.57447800 & -0.77237900\end{array}$

$\begin{array}{lll}8.50375900 & -2.27846100 & -2.36280300\end{array}$

$\begin{array}{lll}8.44165200 & -5.30842200 & -1.52316900\end{array}$

$\begin{array}{lll}11.28718200 & -3.78646900 & -1.78293900\end{array}$

$\begin{array}{lll}7.49384000 & -2.57633900 & -2.68354800\end{array}$

$\begin{array}{lll}9.32855800 & -5.73599300 & -2.01850600\end{array}$

$\begin{array}{lll}8.10240900 & -6.21297800 & -0.32060700\end{array}$

$\begin{array}{lll}7.28917300 & -5.35904900 & -2.54561000\end{array}$

$\begin{array}{lll}11.40665600 & -4.71045300 & 0.14126900\end{array}$

$\begin{array}{lll}11.51616000 & -2.23315300 & -0.32411700\end{array}$

$\begin{array}{lll}8.40916200 & -0.80516000 & -1.92238500\end{array}$

$\begin{array}{lll}9.41490500 & -2.41211000 & -3.60116300\end{array}$

$\begin{array}{lll}11.28469200 & -1.41582000 & -1.01555800\end{array}$

$\begin{array}{llr}11.15066300 & -1.93849000 & 0.66894700\end{array}$

$\begin{array}{lll}12.61078100 & -2.30268800 & -0.25378200\end{array}$

$\begin{array}{lll}12.50501700 & -4.72152300 & 0.18063400\end{array}$

$\begin{array}{lll}11.05201600 & -4.59199200 & 1.17362500\end{array}$

$\begin{array}{llr}11.08271700 & -5.69678500 & -0.20705000\end{array}$

$\begin{array}{rrr}7.20757900 & -5.85344200 & 0.20125000\end{array}$

$\begin{array}{lll}7.90161300 & -7.24216500 & -0.64925100\end{array}$

$\begin{array}{lll}8.91093300 & -6.25303700 & 0.41699500\end{array}$

$\begin{array}{lll}7.51461700 & -4.80563900 & -3.46339000\end{array}$

$\begin{array}{lll}7.07351800 & -6.39714800 & -2.83415900\end{array}$

$\begin{array}{lll}6.35940800 & -4.94882600 & -2.13016900\end{array}$

$\begin{array}{lll}10.43755800 & -2.07896300 & -3.38516000\end{array}$

$\begin{array}{lll}9.47719800 & -3.44468000 & -3.96596700\end{array}$

$\begin{array}{lll}9.04292100 & -1.79400100 & -4.42980500\end{array}$

$\begin{array}{lll}7.68493100 & -0.66214500 & -1.11336900\end{array}$

$\begin{array}{lll}9.37265600 & -0.41662800 & -1.57174500\end{array}$

$\begin{array}{lll}8.09434900 & -0.16903800 & -2.76167000\end{array}$

$\begin{array}{lll}7.39024600 & -1.18026000 & 2.25000500\end{array}$

$\begin{array}{lll}8.29482000 & -0.53395700 & 1.75153700\end{array}$

$\begin{array}{lll}8.53681400 & -1.41062100 & 1.13530500\end{array}$

$\begin{array}{lll}9.00235700 & -0.43080600 & 2.41254900\end{array}$

$\begin{array}{lll}7.56472000 & -3.02691400 & 3.58540100\end{array}$

$\begin{array}{lll}8.59168900 & -2.64733700 & 3.53003600\end{array}$

$\begin{array}{lll}7.18830000 & -2.81176900 & 4.58418500\end{array}$

$\begin{array}{lll}7.62285100 & -4.11559400 & 3.46759400\end{array}$

\section{TS-1a- $\mathrm{CF}_{3} \mathrm{CH}_{2} \mathrm{OH}$}

$\begin{array}{lrrr}\mathrm{C} & -0.53976000 & 0.75974400 & 0.90792300 \\ \mathrm{C} & -0.10854400 & -0.49467300 & 0.48703300 \\ \mathrm{C} & 0.58027700 & -0.59316500 & -0.72591500 \\ \mathrm{C} & 0.82832600 & 0.52945900 & -1.51782500 \\ \mathrm{C} & 0.38395600 & 1.77460800 & -1.07599700\end{array}$




\begin{tabular}{|c|c|c|c|c|c|c|c|}
\hline C & -0.29978700 & 1.91069500 & 0.14002400 & C & -0.70499600 & 3.41485900 & -0.10140000 \\
\hline $\mathrm{H}$ & -1.07630900 & 0.84712100 & 1.84933900 & $\mathrm{H}$ & -0.00031500 & 3.92353100 & 0.56962600 \\
\hline $\mathrm{H}$ & -0.28806400 & -1.38085200 & 1.08352500 & $\mathrm{H}$ & -0.79156200 & 4.02179500 & -1.00795000 \\
\hline $\mathrm{H}$ & 1.34119700 & 0.42229000 & -2.46745900 & $\mathrm{H}$ & -1.67918200 & 3.40654400 & 0.39899600 \\
\hline $\mathrm{H}$ & 0.56634300 & 2.65329300 & -1.68904400 & $\mathrm{~S}$ & 1.24990600 & -2.21372400 & -1.33087000 \\
\hline $\mathrm{C}$ & -0.76362800 & 3.26348800 & 0.62434900 & $\mathrm{O}$ & 0.36050400 & -3.18718600 & -0.71053100 \\
\hline $\mathrm{H}$ & -1.81185800 & 3.23430700 & 0.94306000 & $\mathrm{O}$ & 1.60959700 & -2.28976600 & -2.74878800 \\
\hline $\mathrm{H}$ & -0.17327400 & 3.59445200 & 1.48835700 & $\mathrm{~N}$ & 2.78606400 & -2.31380400 & -0.54419200 \\
\hline $\mathrm{H}$ & -0.66677900 & 4.02475000 & -0.15548100 & $\mathrm{C}$ & 4.00842300 & -2.61302300 & -1.30160600 \\
\hline S & 1.12957300 & -2.18890800 & -1.30123000 & $\mathrm{C}$ & 2.96203100 & -2.31908900 & 0.84583500 \\
\hline $\mathrm{O}$ & 0.22428400 & -3.21889300 & -0.80895600 & $\mathrm{C}$ & 5.03336300 & -2.78267600 & -0.20891800 \\
\hline $\mathrm{O}$ & 1.49660100 & -2.08971700 & -2.71561700 & $\mathrm{H}$ & 3.89049300 & -3.52114000 & -1.90347000 \\
\hline $\mathrm{N}$ & 2.65549900 & -2.40912500 & -0.52430500 & C & 4.41291200 & -2.59104200 & 1.05592100 \\
\hline C & 3.89831100 & -2.56111200 & -1.29881900 & $\mathrm{O}$ & 2.09094900 & -2.14267000 & 1.67592300 \\
\hline $\mathrm{C}$ & 2.83396800 & -2.57621600 & 0.85369200 & $\mathrm{C}$ & 6.37710300 & -3.02739100 & -0.35230000 \\
\hline $\mathrm{C}$ & 4.92408700 & -2.83949600 & -0.22547600 & $\mathrm{C}$ & 7.18761400 & -3.17363100 & 0.79534500 \\
\hline $\mathrm{H}$ & 3.81739600 & -3.38380100 & -2.01738300 & $\mathrm{C}$ & 6.59092900 & -2.95992600 & 2.15812600 \\
\hline $\mathrm{C}$ & 4.27633100 & -2.82940200 & 1.03604200 & $\mathrm{C}$ & 5.14493300 & -2.63888100 & 2.21742100 \\
\hline $\mathrm{O}$ & 1.97443700 & -2.50630100 & 1.71702900 & $\mathrm{H}$ & 6.81747800 & -3.12824600 & -1.33825500 \\
\hline $\mathrm{C}$ & 6.26924800 & -3.08203000 & -0.38179000 & $\mathrm{H}$ & 4.26410300 & -1.79371000 & -1.98309900 \\
\hline C & 7.06113600 & -3.33214100 & 0.76405300 & S & 4.29664500 & -2.31027100 & 3.75098200 \\
\hline $\mathrm{C}$ & 6.42453000 & -3.20483300 & 2.10561000 & $\mathrm{C}$ & 5.48506100 & -1.21217800 & 4.62056400 \\
\hline C & 4.97034400 & -3.05599100 & 2.20469800 & $\mathrm{~F}$ & 4.81520000 & -0.73157900 & 5.67806400 \\
\hline $\mathrm{H}$ & 6.72126300 & -3.09309800 & -1.36733400 & $\mathrm{~F}$ & 6.59413000 & -1.81838400 & 5.08308000 \\
\hline $\mathrm{H}$ & 4.12630500 & -1.64866000 & -1.86139800 & F & 5.88061000 & -0.19114300 & 3.85234700 \\
\hline S & 4.05235000 & -3.08145600 & 3.71218000 & $\mathrm{C}$ & 8.62564800 & -3.23726800 & 0.74711600 \\
\hline $\mathrm{C}$ & 5.35547200 & -3.34824000 & 4.97261400 & $\mathrm{H}$ & 9.03631000 & -3.79704300 & 1.59514000 \\
\hline $\mathrm{F}$ & 4.72271200 & -3.32917000 & 6.15211800 & $\mathrm{Si}$ & 9.68594800 & -3.52441700 & -0.81591700 \\
\hline $\mathrm{F}$ & 5.98494900 & -4.53202000 & 4.85475200 & $\mathrm{C}$ & 11.51089100 & -3.70980700 & -0.23620700 \\
\hline F & 6.29611500 & -2.38986600 & 4.97999100 & $\mathrm{C}$ & 9.49878200 & -2.05997100 & -2.05089200 \\
\hline $\mathrm{C}$ & 8.49492800 & -3.36210400 & 0.78446000 & $\mathrm{C}$ & 9.23048000 & -5.19677300 & -1.69420000 \\
\hline $\mathrm{H}$ & 8.86637800 & -3.93702200 & 1.64404800 & $\mathrm{H}$ & 12.06841600 & -3.90303700 & -1.16709300 \\
\hline $\mathrm{Si}$ & 9.63550300 & -3.56537900 & -0.72919600 & $\mathrm{H}$ & 8.56889300 & -2.25931400 & -2.60684100 \\
\hline $\mathrm{C}$ & 11.43758200 & -3.73388500 & -0.07693200 & $\mathrm{H}$ & 10.20859600 & -5.61091100 & -1.98681100 \\
\hline $\mathrm{C}$ & 9.47358700 & -2.06054600 & -1.91886100 & $\mathrm{C}$ & 8.57149400 & -6.21803900 & -0.74498100 \\
\hline $\mathrm{C}$ & 9.25297100 & -5.20754600 & -1.69299200 & $\mathrm{C}$ & 8.40182000 & -5.07765200 & -2.98852900 \\
\hline $\mathrm{H}$ & 12.03804300 & -3.88384500 & -0.98895000 & $\mathrm{C}$ & 11.74084700 & -4.92729200 & 0.68255700 \\
\hline $\mathrm{H}$ & 8.56851200 & -2.25343300 & -2.51619200 & $\mathrm{C}$ & 12.10990700 & -2.44369900 & 0.40393800 \\
\hline $\mathrm{H}$ & 10.24742100 & -5.58197900 & -1.98439900 & $\mathrm{C}$ & 9.33566300 & -0.67695400 & -1.38705600 \\
\hline $\mathrm{C}$ & 8.60059100 & -6.28771900 & -0.80612900 & $\mathrm{C}$ & 10.63891600 & -2.03743100 & -3.09066600 \\
\hline $\mathrm{C}$ & 8.44356800 & -5.04729900 & -2.99504900 & $\mathrm{H}$ & 12.09280600 & -1.58569800 & -0.27518000 \\
\hline $\mathrm{C}$ & 11.65574400 & -4.97626300 & 0.81050500 & $\mathrm{H}$ & 11.57087400 & -2.14454400 & 1.30900100 \\
\hline $\mathrm{C}$ & 11.98250800 & -2.47792300 & 0.62809100 & $\mathrm{H}$ & 13.15689400 & -2.61446700 & 0.69074700 \\
\hline $\mathrm{C}$ & 9.26596200 & -0.70238600 & -1.21748500 & $\mathrm{H}$ & 12.80666900 & -5.02725400 & 0.92919700 \\
\hline $\mathrm{C}$ & 10.65229200 & -1.99021500 & -2.91231600 & $\mathrm{H}$ & 11.20416600 & -4.82659700 & 1.63509600 \\
\hline $\mathrm{H}$ & 11.97627200 & -1.59906700 & -0.02420000 & $\mathrm{H}$ & 11.42452700 & -5.86909100 & 0.22079200 \\
\hline $\mathrm{H}$ & 11.40024300 & -2.21815300 & 1.51859400 & $\mathrm{H}$ & 7.57373300 & -5.88434400 & -0.43381300 \\
\hline $\mathrm{H}$ & 13.01990300 & -2.63716500 & 0.95385300 & $\mathrm{H}$ & 8.44886600 & -7.19095500 & -1.24035100 \\
\hline $\mathrm{H}$ & 12.71157500 & -5.06074900 & 1.10201900 & $\mathrm{H}$ & 9.15740200 & -6.38322800 & 0.16562700 \\
\hline $\mathrm{H}$ & 11.07407900 & -4.92059100 & 1.74001500 & $\mathrm{H}$ & 8.86523400 & -4.41406500 & -3.72606100 \\
\hline $\mathrm{H}$ & 11.38290900 & -5.90808400 & 0.30338300 & $\mathrm{H}$ & 8.28315500 & -6.06211800 & -3.46162200 \\
\hline $\mathrm{H}$ & 7.58745800 & -5.99448200 & -0.50465800 & $\mathrm{H}$ & 7.38936300 & -4.70071600 & -2.79467300 \\
\hline $\mathrm{H}$ & 8.51607100 & -7.23955600 & -1.34829700 & $\mathrm{H}$ & 11.60216800 & -1.80461700 & -2.62203800 \\
\hline $\mathrm{H}$ & 9.16891000 & -6.47989400 & 0.11027200 & $\mathrm{H}$ & 10.75202600 & -2.99533500 & -3.61355400 \\
\hline $\mathrm{H}$ & 8.90645600 & -4.34474400 & -3.69612300 & $\mathrm{H}$ & 10.45635300 & -1.26795000 & -3.85290100 \\
\hline $\mathrm{H}$ & 8.35014500 & -6.01217200 & -3.51224200 & $\mathrm{H}$ & 8.48118100 & -0.63778200 & -0.70426200 \\
\hline $\mathrm{H}$ & 7.42183200 & -4.69668500 & -2.80038800 & $\mathrm{H}$ & 10.21200900 & -0.39172000 & -0.79630600 \\
\hline $\mathrm{H}$ & 11.59336700 & -1.75794400 & -2.40003400 & $\mathrm{H}$ & 9.19071000 & 0.09863400 & -2.15199800 \\
\hline $\mathrm{H}$ & 10.79907500 & -2.93139100 & -3.45671600 & $\mathrm{H}$ & 7.20532800 & -1.95881100 & 2.36550300 \\
\hline $\mathrm{H}$ & 10.48800600 & -1.20160300 & -3.65907200 & $\mathrm{C}$ & 10.13613400 & 0.53531400 & 2.81220900 \\
\hline $\mathrm{H}$ & 8.38491500 & -0.69672500 & -0.56790900 & $\mathrm{C}$ & 9.25568600 & -0.67093500 & 3.11904900 \\
\hline $\mathrm{H}$ & 10.11608600 & -0.42512600 & -0.58555000 & $\mathrm{H}$ & 9.94776100 & -1.49672800 & 3.37025300 \\
\hline $\mathrm{H}$ & 9.13912800 & 0.09654900 & -1.96141400 & $\mathrm{H}$ & 8.70106500 & -0.42796200 & 4.04039800 \\
\hline $\mathrm{H}$ & 6.97823800 & -2.15906700 & 2.36444000 & $\mathrm{~F}$ & 9.41407900 & 1.64437100 & 2.56856300 \\
\hline $\mathrm{H}$ & 6.86698600 & -3.86288800 & 2.85399500 & $\mathrm{~F}$ & 10.92769400 & 0.32464700 & 1.73323600 \\
\hline $\mathrm{C}$ & 9.81686800 & 0.25720200 & 3.16090800 & $\mathrm{~F}$ & 10.95405400 & 0.80226400 & 3.86095300 \\
\hline $\mathrm{C}$ & 8.94372700 & -0.98344700 & 3.29935300 & $\mathrm{O}$ & 8.41713500 & -0.94246800 & 2.05221600 \\
\hline $\mathrm{H}$ & 9.63027200 & -1.82814200 & 3.48965700 & $\mathrm{H}$ & 8.77279200 & -2.07503800 & 1.24130400 \\
\hline $\mathrm{H}$ & 8.34158200 & -0.84627400 & 4.21178900 & C & 7.08434200 & -3.99176400 & 3.21459500 \\
\hline $\mathrm{F}$ & 9.08661300 & 1.37730800 & 3.01083600 & $\mathrm{H}$ & 8.07938200 & -3.73935400 & 3.58578500 \\
\hline $\mathrm{F}$ & 10.65119200 & 0.17272300 & 2.09858800 & $\mathrm{H}$ & 6.41188700 & -4.03032300 & 4.07032000 \\
\hline $\mathrm{F}$ & 10.58689500 & 0.41512200 & 4.26550000 & $\mathrm{H}$ & 7.12201300 & -4.98963100 & 2.76612700 \\
\hline $\mathrm{O}$ & 8.15670400 & -1.14692500 & 2.16741000 & & & & \\
\hline
\end{tabular}

TS-1a- $\left(\mathrm{H}_{2} \mathrm{O}\right)_{2}$

TS-1n- $\mathrm{CF}_{3} \mathrm{CH}_{2} \mathrm{OH}$

$\begin{array}{lrrr}\mathrm{C} & -0.45733900 & 0.96751100 & 0.49327600 \\ \mathrm{C} & -0.00739100 & -0.32421500 & 0.23420800 \\ \mathrm{C} & 0.68257900 & -0.56550900 & -0.95725600 \\ \mathrm{C} & 0.91337300 & 0.45142600 & -1.88637500 \\ \mathrm{C} & 0.45141800 & 1.73549900 & -1.60509000 \\ \mathrm{C} & -0.23338800 & 2.01506900 & -0.41372700 \\ \mathrm{H} & -0.99578200 & 1.16531900 & 1.41660100 \\ \mathrm{H} & -0.17357300 & -1.12969600 & 0.93891600 \\ \mathrm{H} & 1.42558800 & 0.23228000 & -2.81696900 \\ \mathrm{H} & 0.61976300 & 2.53156800 & -2.32572400\end{array}$

$\begin{array}{lrrr}\mathrm{C} & 0.11882300 & -6.99256600 & -4.04643500 \\ \mathrm{C} & 0.88186300 & -5.83518500 & -4.19706800 \\ \mathrm{C} & 0.66881000 & -4.76827300 & -3.32313500 \\ \mathrm{C} & -0.29434400 & -4.83588500 & -2.31184300 \\ \mathrm{C} & -1.04119800 & -6.00276100 & -2.17870700 \\ \mathrm{C} & -0.85088000 & -7.09507000 & -3.04021100 \\ \mathrm{H} & 0.27650600 & -7.82661200 & -4.72555900 \\ \mathrm{H} & 1.62032200 & -5.74970400 & -4.98682500 \\ \mathrm{H} & -0.43328500 & -3.99827700 & -1.63926000 \\ \mathrm{H} & -1.78842800 & -6.06634200 & -1.39143100 \\ \mathrm{C} & -1.69102700 & -8.34139400 & -2.89303400\end{array}$




\begin{tabular}{|c|c|c|c|c|c|c|c|}
\hline $\mathrm{H}$ & -1.28550200 & -9.17124100 & -3.47991000 & C & 2.68466700 & -3.20156600 & -0.99009800 \\
\hline $\mathrm{H}$ & -1.74985400 & -8.66148800 & -1.84652700 & C & 5.01751800 & -3.24719000 & -1.40399500 \\
\hline $\mathrm{H}$ & -2.71935200 & -8.16505800 & -3.23438100 & $\mathrm{H}$ & 4.58808200 & -4.33977000 & -3.23853900 \\
\hline S & 1.62373800 & -3.27465500 & -3.54155400 & $\mathrm{C}$ & 4.03349200 & -3.13347600 & -0.38170100 \\
\hline $\mathrm{O}$ & 2.32306900 & -3.34808500 & -4.82825400 & $\mathrm{O}$ & 1.57973500 & -3.16640500 & -0.48238900 \\
\hline $\mathrm{O}$ & 0.79431100 & -2.12658900 & -3.19060100 & $\mathrm{C}$ & 6.35497400 & -3.25110600 & -1.14154300 \\
\hline $\mathrm{N}$ & 2.88792900 & -3.40051400 & -2.39580300 & C & 6.85937700 & -3.14946700 & 0.21025800 \\
\hline C & 4.30372000 & -3.40866800 & -2.80842400 & C & 5.83977900 & -2.87143000 & 1.25886900 \\
\hline $\mathrm{C}$ & 2.71630000 & -3.30009000 & -0.99703200 & C & 4.44213700 & -3.00441900 & 0.93758100 \\
\hline $\mathrm{C}$ & 5.02990700 & -3.30539500 & -1.48308800 & $\mathrm{H}$ & 7.06410900 & -3.39177100 & -1.94998600 \\
\hline $\mathrm{H}$ & 4.54734500 & -4.33152300 & -3.34651900 & $\mathrm{H}$ & 4.59637600 & -2.58301000 & -3.44111800 \\
\hline $\mathrm{C}$ & 4.06431200 & -3.24761300 & -0.44270100 & $\mathrm{~S}$ & 3.18348800 & -2.80030000 & 2.22027200 \\
\hline $\mathrm{O}$ & 1.64352700 & -3.27770900 & -0.40877000 & $\mathrm{C}$ & 2.98723100 & -4.54934300 & 2.69433400 \\
\hline $\mathrm{C}$ & 6.37642500 & -3.28683500 & -1.25480100 & F & 2.06159000 & -4.59548700 & 3.66610700 \\
\hline C & 6.88123000 & -3.19665300 & 0.09623400 & F & 2.58050200 & -5.32487700 & 1.68078000 \\
\hline $\mathrm{C}$ & 5.86568800 & -3.01071400 & 1.14806000 & $\mathrm{~F}$ & 4.12417700 & -5.09373500 & 3.17368900 \\
\hline $\mathrm{C}$ & 4.47507000 & -3.16407800 & 0.88386300 & $\mathrm{C}$ & 8.23110200 & -3.21506200 & 0.49457400 \\
\hline $\mathrm{H}$ & 7.07586800 & -3.37468900 & -2.07847300 & $\mathrm{H}$ & 8.46630500 & -3.26591100 & 1.55952200 \\
\hline $\mathrm{H}$ & 4.52102900 & -2.56775100 & -3.47489300 & $\mathrm{Si}$ & 9.65484700 & -3.91166300 & -0.52065800 \\
\hline S & 3.18120300 & -3.14339300 & 2.11374500 & $\mathrm{C}$ & 11.23618700 & -3.51346500 & 0.52136300 \\
\hline $\mathrm{C}$ & 4.11181800 & -3.03422300 & 3.67972000 & C & 9.75297500 & -3.23557100 & -2.32830200 \\
\hline $\mathrm{F}$ & 3.18951700 & -3.01161500 & 4.65421700 & C & 9.51425200 & -5.83544600 & -0.62520000 \\
\hline $\mathrm{F}$ & 4.93028500 & -4.07784900 & 3.90571500 & $\mathrm{H}$ & 10.91190000 & -3.70937000 & 1.55686800 \\
\hline $\mathrm{F}$ & 4.85676900 & -1.91557100 & 3.78845700 & $\mathrm{H}$ & 8.93417900 & -3.73981400 & -2.86552100 \\
\hline $\mathrm{C}$ & 8.24925500 & -3.17319700 & 0.42085300 & $\mathrm{H}$ & 10.45582300 & -6.19144400 & -1.07200300 \\
\hline $\mathrm{H}$ & 8.42335800 & -3.20911500 & 1.50395100 & $\mathrm{C}$ & 9.39038500 & -6.47571500 & 0.77224800 \\
\hline $\mathrm{Si}$ & 9.72809100 & -3.86066000 & -0.51481400 & $\mathrm{C}$ & 8.36519400 & -6.32832000 & -1.52546000 \\
\hline $\mathrm{C}$ & 11.27571500 & -3.30208500 & 0.50874200 & $\mathrm{C}$ & 11.68898000 & -2.04045100 & 0.46564000 \\
\hline $\mathrm{C}$ & 9.81289000 & -3.31183300 & -2.36329300 & $\mathrm{C}$ & 12.44487300 & -4.43333000 & 0.25380300 \\
\hline $\mathrm{C}$ & 9.68332400 & -5.79173800 & -0.48400800 & $\mathrm{C}$ & 9.51931700 & -1.71960600 & -2.47999400 \\
\hline $\mathrm{H}$ & 10.93773800 & -3.38651100 & 1.55501000 & $\mathrm{C}$ & 11.05448800 & -3.64817300 & -3.04785600 \\
\hline $\mathrm{H}$ & 8.99084700 & -3.85726300 & -2.85267600 & $\mathrm{H}$ & 12.19928300 & -5.49244700 & 0.38098900 \\
\hline $\mathrm{H}$ & 10.65846300 & -6.13442700 & -0.86371400 & $\mathrm{H}$ & 12.83669800 & -4.30632900 & -0.76216900 \\
\hline $\mathrm{C}$ & 9.52284500 & -6.33424200 & 0.95015400 & $\mathrm{H}$ & 13.26712700 & -4.20317700 & 0.94599200 \\
\hline $\mathrm{C}$ & 8.59623700 & -6.39345700 & -1.39544500 & $\mathrm{H}$ & 12.47130800 & -1.84184100 & 1.21170600 \\
\hline $\mathrm{C}$ & 11.70120900 & -1.83583800 & 0.28857300 & $\mathrm{H}$ & 12.11175500 & -1.78763300 & -0.51422500 \\
\hline $\mathrm{C}$ & 12.51225500 & -4.21361100 & 0.36681800 & $\mathrm{H}$ & 10.86950100 & -1.33718100 & 0.65090100 \\
\hline $\mathrm{C}$ & 9.57449100 & -1.81092600 & -2.61883300 & $\mathrm{H}$ & 8.47095100 & -6.14880600 & 1.27335800 \\
\hline $\mathrm{C}$ & 11.11066900 & -3.77333400 & -3.05904300 & $\mathrm{H}$ & 9.35242500 & -7.57161600 & 0.70070800 \\
\hline $\mathrm{H}$ & 12.29679300 & -5.25477000 & 0.62697700 & $\mathrm{H}$ & 10.23148900 & -6.22084500 & 1.42810000 \\
\hline $\mathrm{H}$ & 12.90782700 & -4.20496900 & -0.65579500 & $\mathrm{H}$ & 8.46281900 & -5.97563500 & -2.55884400 \\
\hline $\mathrm{H}$ & 13.32219800 & -3.87259500 & 1.02706100 & $\mathrm{H}$ & 8.34049200 & -7.42658400 & -1.55931400 \\
\hline $\mathrm{H}$ & 12.45925000 & -1.53347600 & 1.02498300 & $\mathrm{H}$ & 7.39015500 & -5.99574900 & -1.14893700 \\
\hline $\mathrm{H}$ & 12.14762400 & -1.69632100 & -0.70323800 & $\mathrm{H}$ & 11.92898500 & -3.15125800 & -2.61141000 \\
\hline $\mathrm{H}$ & 10.86704900 & -1.12899200 & 0.36269500 & $\mathrm{H}$ & 11.23209900 & -4.72896200 & -3.00271000 \\
\hline $\mathrm{H}$ & 8.55701200 & -6.03649700 & 1.37599600 & $\mathrm{H}$ & 11.01972900 & -3.36514300 & -4.10904200 \\
\hline $\mathrm{H}$ & 9.56234500 & -7.43237600 & 0.96349700 & $\mathrm{H}$ & 8.56401200 & -1.40097300 & -2.04856800 \\
\hline $\mathrm{H}$ & 10.30583300 & -5.97060700 & 1.62655000 & $\mathrm{H}$ & 10.30525900 & -1.13409200 & -1.99044200 \\
\hline $\mathrm{H}$ & 8.73511800 & -6.11869200 & -2.44785800 & $\mathrm{H}$ & 9.51533200 & -1.43189700 & -3.54084500 \\
\hline $\mathrm{H}$ & 8.60533600 & -7.49133100 & -1.34252000 & $\mathrm{H}$ & 8.91301800 & -0.14876500 & 1.48176000 \\
\hline $\mathrm{H}$ & 7.59373700 & -6.06448100 & -1.09460300 & $\mathrm{O}$ & 8.41712600 & -0.32770900 & 0.66583400 \\
\hline $\mathrm{H}$ & 11.98758500 & -3.24706400 & -2.66318400 & $\mathrm{H}$ & 8.48372500 & -1.33393000 & 0.51701100 \\
\hline $\mathrm{H}$ & 11.28885000 & -4.84828900 & -2.93852700 & $\mathrm{H}$ & 6.97850000 & -0.14203800 & 0.81531600 \\
\hline $\mathrm{H}$ & 11.07149600 & -3.56579600 & -4.13742800 & $\mathrm{O}$ & 5.92383100 & -0.22006500 & 0.90138900 \\
\hline $\mathrm{H}$ & 8.63291400 & -1.45660400 & -2.18554200 & $\mathrm{H}$ & 5.77278600 & -1.36837000 & 1.03163100 \\
\hline $\mathrm{H}$ & 10.37574900 & -1.19687400 & -2.19373300 & $\mathrm{H}$ & 5.53400600 & 0.01760100 & 0.03955000 \\
\hline $\mathrm{H}$ & 9.54361200 & -1.60013400 & -3.69721600 & C & 6.32079400 & -2.90571800 & 2.70105600 \\
\hline $\mathrm{H}$ & 8.96897100 & -0.12750000 & 1.23580100 & $\mathrm{H}$ & 7.13095400 & -2.18445600 & 2.86606400 \\
\hline $\mathrm{H}$ & 6.22011900 & -3.16180600 & 2.16268800 & $\mathrm{H}$ & 5.51774600 & -2.67448500 & 3.40016200 \\
\hline $\mathrm{O}$ & 8.35622800 & -0.33817200 & 0.51148100 & $\mathrm{H}$ & 6.71953300 & -3.89637900 & 2.95467000 \\
\hline $\mathrm{H}$ & 8.43687700 & -1.35892300 & 0.36805100 & & & & \\
\hline $\mathrm{H}$ & 7.01390200 & -0.22477800 & 0.91571000 & & & & \\
\hline $\mathrm{O}$ & 5.97605600 & -0.33464100 & 1.20292300 & & & & \\
\hline $\mathrm{H}$ & 5.83281500 & -1.46419100 & 1.18994300 & & & & \\
\hline $\mathrm{H}$ & 5.41620800 & 0.03232400 & 0.49387600 & & & & \\
\hline
\end{tabular}

TS-1n-(H2O)

$\begin{array}{lrrr}\mathrm{C} & 0.32955100 & -7.11298400 & -3.89543600 \\ \mathrm{C} & 1.06083700 & -5.94224400 & -4.08762900 \\ \mathrm{C} & 0.77063300 & -4.82730900 & -3.29864000 \\ \mathrm{C} & -0.24021500 & -4.86142900 & -2.33416100 \\ \mathrm{C} & -0.95545200 & -6.04324100 & -2.15824100 \\ \mathrm{C} & -0.68259600 & -7.18445700 & -2.92796600 \\ \mathrm{H} & 0.54430900 & -7.98240900 & -4.51183200 \\ \mathrm{H} & 1.83047000 & -5.88098400 & -4.84934300 \\ \mathrm{H} & -0.44465700 & -3.98460900 & -1.73231200 \\ \mathrm{H} & -1.74352700 & -6.07874500 & -1.41020900 \\ \mathrm{C} & -1.45080400 & -8.46517000 & -2.70374500 \\ \mathrm{H} & -1.42666400 & -9.10868200 & -3.58898100 \\ \mathrm{H} & -1.02339800 & -9.03754300 & -1.86973700 \\ \mathrm{H} & -2.49860200 & -8.26526600 & -2.45535500 \\ \mathrm{~S} & 1.69118100 & -3.32087600 & -3.57157100 \\ \mathrm{O} & 2.43828300 & -3.45113500 & -4.82701900 \\ \mathrm{O} & 0.82175200 & -2.17684000 & -3.32016900 \\ \mathrm{~N} & 2.91981100 & -3.34163900 & -2.37978300 \\ \mathrm{C} & 4.34077700 & -3.39138800 & -2.74796700\end{array}$

